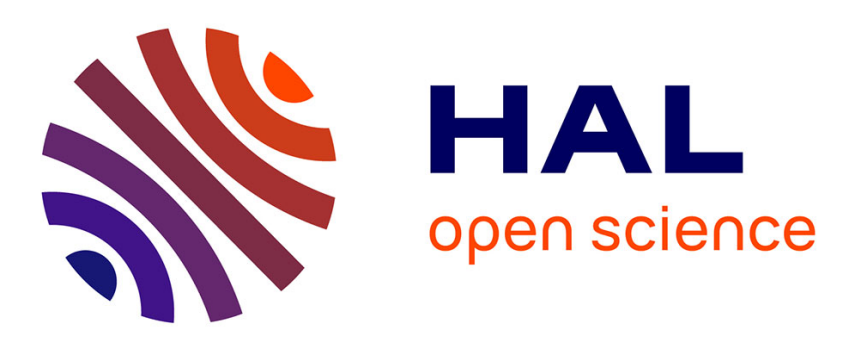

\title{
Surface and interface acoustic waves in solid-fluid superlattices: Green's function approach
}

Y. El Hassouani, E.H. El Boudouti, Bahram Djafari-Rouhani, H. Aynaou, L. Dobrzynski

\section{- To cite this version:}

Y. El Hassouani, E.H. El Boudouti, Bahram Djafari-Rouhani, H. Aynaou, L. Dobrzynski. Surface and interface acoustic waves in solid-fluid superlattices: Green's function approach. Physical Review B: Condensed Matter and Materials Physics (1998-2015), 2006, 74 (14), pp.144306. 10.1103/PhysRevB.74.144306 . hal-00154926

\section{HAL Id: hal-00154926 \\ https://hal.science/hal-00154926}

Submitted on 23 Aug 2021

HAL is a multi-disciplinary open access archive for the deposit and dissemination of scientific research documents, whether they are published or not. The documents may come from teaching and research institutions in France or abroad, or from public or private research centers.
L'archive ouverte pluridisciplinaire HAL, est destinée au dépôt et à la diffusion de documents scientifiques de niveau recherche, publiés ou non, émanant des établissements d'enseignement et de recherche français ou étrangers, des laboratoires publics ou privés. 


\title{
Surface and interface acoustic waves in solid-fluid superlattices: Green's function approach
}

\author{
Y. El Hassouani, ${ }^{1}$ E. H. El Boudouti, ${ }^{1}$ B. Djafari-Rouhani, ${ }^{2}$ H. Aynaou, ${ }^{1}$ and L. Dobrzynski ${ }^{2}$ \\ ${ }^{1}$ Laboratoire de Dynamique et d'Optique des Matériaux, Département de Physique, Faculté des Sciences, \\ Université Mohamed Premier, 60000 Oujda, Morocco \\ ${ }^{2}$ Institut d'Electronique, de Microélectronique et de Nanotechnologie (IEMN), UMR CNRS 8520 and UFR de Physique, \\ Université de Lille 1, 59655 Villeneuve d'Ascq, France
}

(Received 14 June 2006; revised manuscript received 28 August 2006; published 20 October 2006)

\begin{abstract}
We study the propagation of acoustic waves associated with the surface of a semi-infinite superlattice (SL) consisting of alternating elastic solid and ideal fluid layers or its interface with a semi-infinite fluid. We present closed-form expressions for localized surface and interface waves depending on whether the SL is terminated with a fluid layer or a solid layer. We also calculate the corresponding Green's function and densities of states. These general results are illustrated by a few applications to periodic Plexiglas-water and Al-water SLs. In the case of a fluid layer termination, we generalize a rule obtained previously about the existence and behavior of surface waves in the case of pure transverse or longitudinal waves in solid-solid SLs, namely (i) the creation from the infinite SL of a free surface gives rise to $\delta$ peaks of weight $(-1 / 4)$ in the density of states, at the edges of the SL bulk bands, (ii) by considering together the two complementary semi-infinite SLs obtained by the cleavage of an infinite SL along a plane lying within the fluid layer and parallel to the interfaces, one always has as many localized surface modes as minigaps, for any value of the wave vector $k_{\|}$(parallel to the interfaces). However, this rule is not fulfilled when the cleavage is carried out inside the solid layer. Indeed, in this case, the dispersion curves may present zero, one, or two modes inside each gap of the two complementary SLs depending on the position of the plane where the cleavage is produced. Finally, we investigate the localized and resonant modes associated with the presence of a fluid cap layer made of mercury, with finite or semi-infinite extent, on top of the above-mentioned SLs. Different guided modes induced by the adsorbed fluid layer are obtained and their properties are investigated.
\end{abstract}

DOI: 10.1103/PhysRevB.74.144306

PACS number(s): 62.30. $+\mathrm{d}, 68.35 . \mathrm{Iv}, 43.20 .+\mathrm{g}$

\section{INTRODUCTION}

During the past two decades, much attention has been devoted to the study of wave propagation in one-dimensional (1D) periodically stratified media in many contexts, including acoustic, elastic, and electromagnetic systems. ${ }^{1,2}$ The essential property of these structures is the existence of forbidden frequency bands induced by the difference in acoustic and dielectric properties of the constituents and the periodicity of these systems. With regard to acoustic waves, a number of theoretical and experimental works have been devoted to the study of the band-gap structures of periodic solid-solid $\mathrm{SLs}^{3-6}$ composed of crystalline, amorphous semiconductors or metallic multilayers at the nanometric scale. The theoretical models used are essentially the transfer matrix ${ }^{3,7}$ and the Green's function methods, ${ }^{8,9}$ whereas the experimental techniques include phonon spectroscopy, ${ }^{10-14}$ Raman scattering, ${ }^{15-17}$ ultrasonics, ${ }^{18-21}$ and time-resolved x-ray diffraction. ${ }^{22}$ Besides the existence of the band-gap structures in perfect periodic SLs, it was shown theoretically and experimentally that the introduction of inhomogeneities such as a free surface, ${ }^{8,23-30}$ a superlattice/substrate interface, ${ }^{8,26,31,32}$ and a cavity laye ${ }^{33-39}$ within such SLs may induce defect modes inside the gaps. Recently, ${ }^{40,41}$ it was shown that periodic lamellar structures may exhibit the property of omnidirectional reflection for acoustic waves (acoustic mirror), i.e., it reflects any acoustic wave independent of its polarization and incidence angle in analogy with 2D and 3D phononic crystals. ${ }^{42}$ Also, these structures may be used as acoustic filters if a defect layer (cavity) is introduced in the perfect structure. ${ }^{43}$
Wave propagation in alternating elastic solid and ideal fluid layers is carried out by Rytov ${ }^{44}$ and summarized by Brekhovskikh. ${ }^{1}$ Rytov's approach has been used by Schoenberg ${ }^{45}$ together with the propagator matrix formalism to account for propagation through such a periodic medium in any direction of propagation and at arbitrary frequency. Similar results are also obtained by Rousseau. ${ }^{46}$ In the lowfrequency limit, it was shown that besides the existence of small gaps, there is one wave speed for propagation perpendicular to the layering and two wave speeds for propagation parallel to the layering that are without analogue in solidsolid SLs. The two latter speeds both correspond to compressional waves, and their existence is suggestive of Biot's theory ${ }^{47}$ of wave propagation in porous media. The experimental evidence ${ }^{48}$ of these waves is carried out using ultrasonic techniques in Al-water and Plexiglas-water SLs. Also, it was shown theoretically and experimentally that finite-size layered structures composed of a few cells of solid-fluid layers with one $e^{49,50}$ or multiple ${ }^{51}$ periodicity may exhibit large gaps, and the presence of defect layers in these structures may give rise to well defined defect modes in these gaps. ${ }^{50}$

However, to our knowledge, in all of these studies the possibility of the existence of surface and interface waves associated with the surface of a semi-infinite solid-fluid SL or its interface with a homogeneous fluid has not been addressed. In this paper, we give explicit dispersion relations for surface acoustic waves in semi-infinite solid-fluid SLs with a surface cap layer. The cap layer may be a fluid layer or a solid layer different from those constituting the bulk SL. When the thickness of the cap layer goes to zero or to infinity, we obtain the results for a SL with a free surface or for 
the interface between a SL and a homogeneous medium, respectively. These investigations are done within the framework of the response function associated with such heterostructures. ${ }^{52}$ The knowledge of these Green's functions enables us to calculate also the local and total density of states. Then in addition to the dispersion of extended and localized states, one can also obtain the spatial distribution of the modes and, in particular, the possibility of resonant modes (leaky waves), which may appear as well-defined peaks of the density of states (DOS) inside the bulk bands.

The organization of this paper is as follows. Section II presents the model we use for these studies as well as the analytical results obtained for the dispersion relations of bulk and surface waves in the above-discussed heterostructures. The analytical expressions of local and total DOS are rather complicated, therefore we shall present a brief summary of the method of calculation of these DOS. The expressions of the Green's functions necessary for all these studies are given in the Appendix. Section III contains the numerical results for Plexiglas-water and Al-water semi-infinite SLs with or without a surface cap layer and for such semi-infinite SLs in contact with a homogeneous fluid. The main conclusions are summarized in Sec. IV.

\section{THE THEORETICAL MODEL}

\section{A. Interface response theory of continuous media}

Our theoretical analysis is performed with the help of the interface response theory of continuous media, ${ }^{52}$ which allows us to calculate the Green's function of any composite material. In what follows, we present the basic concept and the fundamental equations of this theory. ${ }^{52}$ Let us consider any composite material contained in its space of definition $D$ and formed out of $N$ different homogeneous pieces located in their domains $D_{i}$. Each piece is bounded by an interface $M_{i}$, adjacent in general to $j(1 \leqslant j \leqslant J)$ other pieces through subinterface domains $M_{i j}$. The ensemble of all these interface spaces $M_{i}$ will be called the interface space $M$ of the composite material. The elements of the Green's function $g(D D)$ of any composite material can be obtained from ${ }^{52}$

$$
\begin{aligned}
g(D D)= & G(D D)-G(D M) G^{-1}(M M) G(M D) \\
& +G(D M) G^{-1}(M M) g(M M) G^{-1}(M M) G(M D),
\end{aligned}
$$

where $G(D D)$ is the reference Green's function formed out of truncated pieces in $D_{i}$ of the bulk Green's functions of the infinite continuous media and $g(M M)$, the interface element of the Green's function of the composite system. The inverse of $g(M M)$ is obtained as a superposition of the different $\left[g_{i}\left(M_{i}, M_{i}\right)\right]^{-1}$, where $g_{i}\left(M_{i}, M_{i}\right)$ is the interface Green's function for each constituent $i$ of the composite system. ${ }^{52}$ The knowledge of the inverse of $g(M M)$ is sufficient to calculate the interface states of a composite system through the relation $^{52}$

$$
\operatorname{det}\left[g^{-1}(M M)\right]=0 .
$$

In this paper, we are interested in the propagation of acoustic waves polarized in the sagittal plane defined by the normal to the interfaces ( $z$ direction) and the wave vector $k_{\|}$ (parallel to the interfaces). We choose $k_{\|}$along the $x$ direction. Before addressing the problem of the fluid-solid SL, it is helpful to know the surface elements of its elementary constituents, namely the Green's function of an ideal fluid of thickness $d_{f}$, sound speed $v_{f}$, and mass density $\rho_{f}$ and an elastic isotropic solid characterized by its thickness $d_{s}$, longitudinal speed $v_{\ell}$, transverse speed $v_{t}$, and mass density $\rho_{s}$. The Green's function of the elastic solid in the space of the two surfaces of the layer is a $4 \times 4$ matrix (see the Appendix $)^{53,54}$ as it exhibits two directions of vibrations, while the Green's function of an ideal fluid (for which the viscous shear stress vanishes) is a $4 \times 4$ matrix where only $z z$ components are different from zero (see the Appendix). ${ }^{53,54}$ Therefore, the $4 \times 4$ matrix of the fluid layer may be reduced to only a $2 \times 2$ matrix composed by the $z z$ elements. We shall call this matrix

$$
\left[g_{f}(M M)\right]^{-1}=\left(\begin{array}{ll}
a & b \\
b & a
\end{array}\right)
$$

where

$$
\begin{gathered}
a=-F \frac{C_{f}}{S_{f}}, \quad b=\frac{F}{S_{f}}, \quad C_{f}=\cosh \left(\alpha_{f} d_{f}\right), \quad S_{f}=\sinh \left(\alpha_{f} d_{f}\right), \\
F=-\rho_{f} \frac{\omega^{2}}{\alpha_{f}}, \quad \text { and } \quad \alpha_{f}^{2}=k_{\|}^{2}-\frac{\omega^{2}}{v_{f}^{2}} .
\end{gathered}
$$

It is worthwhile to notice that the assumption of ideal fluid behavior is valid over a very broad frequency range for which the viscous skin depth $\sigma=(2 \eta / \rho \omega)$ is much smaller than the fluid layer thickness $d_{f}$ ( $\eta$ is the viscosity of the fluid).

As concerns the elastic layer, its $4 \times 4$ matrix may also be reduced to a $2 \times 2$ matrix as far as it is surrounded by fluids on both sides. This is due to the fact that the shear stress vanishes on both surfaces. The reduction of the $4 \times 4$ matrix of the elastic solid must be done carefully by inverting the $4 \times 4$ matrix given in the Appendix and keeping only the $z z$ components of this matrix to form a $2 \times 2$ matrix. Then by inverting again this matrix, we obtain a $2 \times 2$ Green's function matrix of the solid layer that may be juxtaposed to the $2 \times 2$ matrix of the fluid layer to form the SL. After some algebraic calculations, the expression of this matrix can be written in a closed form as

$$
\left[g_{s}(M M)\right]^{-1}=\left(\begin{array}{cc}
A & B \\
B & A
\end{array}\right)
$$

where

$$
\begin{gathered}
A=-\gamma \frac{C_{\ell}}{S_{\ell}}-\beta \frac{C_{t}}{S_{t}}, \quad B=\frac{\gamma}{S_{\ell}}+\frac{\beta}{S_{t}}, \\
\gamma=-\rho_{s} \frac{v_{t}^{4}}{\omega^{2} \alpha_{\ell}}\left(k_{\|}^{2}+\alpha_{t}^{2}\right)^{2}, \quad \beta=4 \rho_{s} \frac{v_{t}^{4}}{\omega^{2}} \alpha_{t} k_{\|}^{2},
\end{gathered}
$$




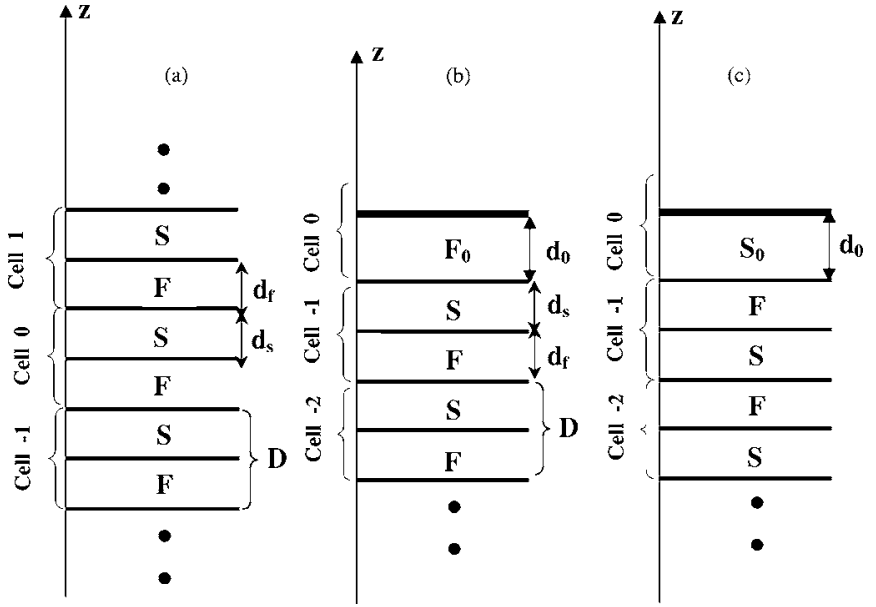

FIG. 1. (a) Schematic representation of an infinite solid/fluid superlattice (SL). (b) Schematic representation of a semi-infinite solid/fluid SL with a fluid cap layer. (c) Schematic representation of a semi-infinite solid/fluid SL with a solid cap layer. $d_{s}, d_{f}$, and $d_{0}$ are, respectively, the thicknesses of the solid, fluid, and cap layers. $D=d_{f}+d_{s}$ is the period of the SL.

$$
\begin{array}{cc}
C_{t}=\cosh \left(\alpha_{t} d_{s}\right), & C_{\ell}=\cosh \left(\alpha_{\ell} d_{s}\right), \\
S_{t}=\sinh \left(\alpha_{t} d_{s}\right), & S_{\ell}=\sinh \left(\alpha_{\ell} d_{s}\right),
\end{array}
$$

and

$$
\alpha_{t}^{2}=k_{\|}^{2}-\frac{\omega^{2}}{v_{t}^{2}}, \quad \alpha_{\ell}^{2}=k_{\|}^{2}-\frac{\omega^{2}}{v_{\ell}^{2}} .
$$

Now, the calculation of the dispersion relations of an infinite SL or a semi-infinite SL with or without a surface cap layer becomes analogous to that of shear horizontal waves in solid-solid $\mathrm{SLs}^{8}$ including only one degree of vibration. The effect of the shear waves in solid layers implicitly appears in the terms $A$ and $B$ [Eq. (5)]. Indeed, if the transverse velocity of sound in the solid layer vanishes [i.e., $v_{t}=0$ in Eqs. (5)-(8)], one obtains the results for a SL made of alternating two different fluids.

\section{B. Dispersion relations}

The Green's function of the infinite SL [Fig. 1(a)] is obtained by a linear juxtaposition of the $2 \times 2$ matrices [Eqs. (3) and (5)] at the different interfaces, leading to a tridiagonal matrix. After a Fourier transform, we obtain the following expression giving the dispersion relation of an infinite SL:

$$
\cos \left(k_{z} D\right)=\frac{A^{2}-B^{2}+a^{2}-b^{2}+2 A a}{B b},
$$

where $k_{z}$ is the component perpendicular to the slabs of the propagation vector $\vec{k} \equiv\left(k_{\|}, k_{z}\right)$.

In the same way, the dispersion relation giving the surface modes for a semi-infinite SL terminated with a fluid cap layer characterized by its mass density $\rho_{0}$, sound speed $v_{0}$, and thickness $d_{0}$ [Fig. 1(b)] is given by

$$
\begin{gathered}
a\left(B^{2}-A^{2}\right)-A\left(a^{2}-b^{2}\right)-\frac{f_{0} S_{0}}{C_{0}}\left[A^{2}-B^{2}-a^{2}+b^{2}\right] \\
+\left(\frac{f_{0} S_{0}}{C_{0}}\right)^{2}(A+a)=0
\end{gathered}
$$

together with the following condition:

$$
\left|\frac{B}{b}\left(\frac{a+\frac{f_{0} S_{0}}{C_{0}}}{A-\frac{f_{0} S_{0}}{C_{0}}}\right)\right|>1,
$$

where

$$
\begin{aligned}
& C_{0}=\cosh \left(\alpha_{0} d_{0}\right), \quad S_{0}=\sinh \left(\alpha_{0} d_{0}\right), \\
& f_{0}=-\rho_{0} \frac{\omega^{2}}{\alpha_{0}}, \quad \text { and } \quad \alpha_{0}^{2}=k_{\|}^{2}-\frac{\omega^{2}}{v_{0}^{2}} .
\end{aligned}
$$

The latter condition [Eq. (11)] ensures that the waves are decaying from the surface when penetrating into the SL.

From these general expressions, we can deduce the following:

(i) The expressions giving the surface modes for a semiinfinite SL without a cap layer (i.e., $d_{0}=0, S_{0}=0$ ),

$$
a\left(B^{2}-A^{2}\right)-A\left(a^{2}-b^{2}\right)=0,
$$

with the condition

$$
\left|\frac{a B}{A b}\right|>1
$$

(ii) The expressions giving the interface modes between a SL and a homogeneous fluid of a semi-infinite extent (i.e., $\left.d_{0} \rightarrow \infty, \frac{S_{0}}{C_{0}} \rightarrow 1\right)$,

$$
\begin{aligned}
& a\left(B^{2}-A^{2}\right)-A\left(a^{2}-b^{2}\right)-f_{0}\left[A^{2}-B^{2}-a^{2}+b^{2}\right]+f_{0}^{2}(A+a) \\
& \quad=0,
\end{aligned}
$$

with the condition

$$
\left|\frac{B}{b}\left(\frac{a+f_{0}}{A-f_{0}}\right)\right|>1 .
$$

When the SL is terminated with a solid cap layer [Fig. 1(c)] characterized by its thickness $d_{0}$, mass density $\rho_{0}$, and transverse and longitudinal sound speeds $v_{t 0}$ and $v_{\ell 0}$, respectively, the expression giving the surface modes is given by

$$
\begin{aligned}
& a\left(B^{2}-A^{2}\right)-A\left(a^{2}-b^{2}\right)-F_{0}\left[A^{2}-B^{2}-a^{2}+b^{2}\right]+F_{0}^{2}(A+a) \\
& \quad=0
\end{aligned}
$$

together with the condition

$$
\left|\frac{b}{B}\left(\frac{A-F_{0}}{a+F_{0}}\right)\right|>1,
$$

where

$$
F_{0}=\frac{A_{0}^{2}-B_{0}^{2}}{A_{0}},
$$




$$
\begin{gathered}
A_{0}=-\gamma_{0} \frac{C_{\ell 0}}{S_{\ell 0}}-\beta_{0} \frac{C_{t 0}}{S_{t 0}}, \quad B_{0}=\frac{\gamma_{0}}{S_{\ell 0}}+\frac{\beta_{0}}{S_{t 0}}, \\
\gamma_{0}=-\rho_{0} \frac{v_{t 0}^{4}}{\omega^{2} \alpha_{\ell 0}}\left(k_{\|}^{2}+\alpha_{t 0}^{2}\right)^{2}, \quad \beta_{0}=4 \rho_{0} \frac{v_{t 0}^{4}}{\omega^{2}} \alpha_{t 0} k_{\|}^{2}, \\
C_{t 0}=\cosh \left(\alpha_{t 0} d_{0}\right), \quad C_{\ell 0}=\cosh \left(\alpha_{\ell 0} d_{0}\right), \\
S_{t 0}=\sinh \left(\alpha_{t 0} d_{0}\right), \quad S_{\ell 0}=\sinh \left(\alpha_{\ell 0} d_{0}\right),
\end{gathered}
$$

and

$$
\alpha_{t 0}^{2}=k_{\|}^{2}-\frac{\omega^{2}}{v_{t 0}^{2}}, \quad \alpha_{\ell 0}^{2}=k_{\|}^{2}-\frac{\omega^{2}}{v_{\ell 0}^{2}} .
$$

As before, from these general expressions, we can obtain the following:

(i) The expressions giving the surface modes for a semiinfinite SL without a cap layer (i.e., $d_{0}=0, S_{t 0}=0$, and $S_{\ell 0}$ $=0)$,

$$
a\left(B^{2}-A^{2}\right)-A\left(a^{2}-b^{2}\right)=0,
$$

with

$$
\left|\frac{A b}{a B}\right|>1
$$

(ii) The expressions giving the interface modes between a SL and a homogeneous solid of a semi-infinite extent (i.e., $d_{0} \rightarrow \infty, \frac{S_{t 0}}{C_{t 0}} \rightarrow 1$, and $\left.\frac{S_{\ell 0}}{C_{\ell 0}} \rightarrow 1\right)$. These expressions are the same as in Eqs. (17) and (18) with $F_{0}$ [Eq. (19)] given by $F_{0}$ $=-\left(\gamma_{0}+\beta_{0}\right)$.

It is worth mentioning that the expressions giving the surface states for a semi-infinite SL ended with a full solid layer [Eq. (13)] or a full fluid layer [Eq. (23)] are exactly the same, however the conditions ensuring the decaying of these surface modes [Eqs. (14) and (24)] are different in both cases. In particular, we can notice that these two conditions are the inverse of each other, which means that if a surface mode exists on one SL, it does not exist on the surface of the complementary SL. More interestingly, it can be shown easily from Eqs. (2), (3), and (5) that the expression giving the surface waves for the two complementary semi-infinite SLs is exactly the same expression giving the standing waves of a fluid-solid bilayer with stress-free boundary conditions.

\section{Densities of states}

The calculation of the densities of states can be carried out from the calculation of the Green's function for the infinite and semi-infinite SLs described in Fig. 1. This can be done using Eq. (1) and the Green's functions given in the Appendix for the infinite continuous media and the layered media. The details of the analysis are the same as for shear horizontal waves ${ }^{8}$ but with more complicated calculations. Let us emphasize that in the geometry of the studied structures, all the interfaces are taken to be parallel to the $(x, y)$ plane. A space position along the $z$ axis in medium $i$ belonging to the unit cell $n$ is indicated by $(n, i, z)$, where $-d_{i} / 2$ $<z<d_{i} / 2$ ( $i=f$ for the fluid and $i=s$ for the solid, see Fig. 1). As we are interested in the propagation of sagittal acoustic waves in such structures, the elements of the Green's functions take the form $g_{\alpha \beta}\left(\omega^{2}, k_{\|} \mid n, i, z ; n^{\prime}, i^{\prime}, z^{\prime}\right)$, where $\omega$ is the frequency of the acoustic wave, $k_{\|}$is the wave vector parallel to the interfaces, and $\alpha, \beta$ denote the directions $x$ and $z$. For the sake of simplicity, we shall omit in the following the parameters $\omega^{2}$ and $k_{\|}$, and we note as $g\left(n, i, z ; n^{\prime}, i^{\prime}, z^{\prime}\right)$ the $2 \times 2$ matrix whose elements are $g_{\alpha \beta}\left(n, i, z ; n^{\prime}, i^{\prime}, z^{\prime}\right)$ $(\alpha, \beta=x, z)$. From these Green's functions, one obtains for a given value of $k_{\|}$the local density of states,

$$
n_{\alpha}\left(\omega^{2}, k_{\|} ; n, i, z\right)=-\frac{1}{\pi} \operatorname{Im} g_{\alpha \alpha}\left(\omega^{2}, k_{\|} \mid n, i, z ; n, i, z\right)(\alpha=x, z),
$$

or equivalently

$$
n_{\alpha}\left(\omega, k_{\|} ; n, i, z\right)=-\frac{2 \omega}{\pi} \operatorname{Im} g_{\alpha \alpha}\left(\omega^{2}, k_{\|} \mid n, i, z ; n, i, z\right)(\alpha=x, z)
$$

The total density of states for a given value of $k_{\|}$is obtained by integrating over $z$ and summing over $n, i$, and $\alpha$ the local density of states from which the contributions of the infinite SL have been subtracted. This variation $\Delta n(\omega)$ can be written as the sums of the variations $\Delta n_{f}(\omega)$ and $\Delta n_{s}(\omega)$ in the DOS in the fluid and solid layers, and the DOS $n_{0}(\omega)$ in the cap layer, respectively,

$$
\Delta n\left(\omega^{2}\right)=\Delta n_{f}\left(\omega^{2}\right)+\Delta n_{s}\left(\omega^{2}\right)+n_{0}\left(\omega^{2}\right),
$$

where

$$
\begin{aligned}
\Delta n_{f}\left(\omega^{2}\right)= & -\frac{\rho_{f}}{\pi} \operatorname{Im} \operatorname{tr} \sum \int[\mathbf{d}(n, i=f, z ; n, i=f, z) \\
& -\mathbf{g}(n, i=f, z ; n, i=f, z)] d z, \\
\Delta n_{s}\left(\omega^{2}\right)= & -\frac{\rho_{s}}{\pi} \operatorname{Im} \operatorname{tr} \sum \int[\mathbf{d}(n, i=s, z ; n, i=s, z) \\
& -\mathbf{g}(n, i=s, z ; n, i=s, z)] d z, \\
n_{0}\left(\omega^{2}\right)= & -\frac{\rho_{0}}{\pi} \operatorname{Im} \operatorname{tr} \int \mathbf{d}(n, i=0, z ; n, i=0, z) d z,
\end{aligned}
$$

where $\mathbf{d}$ and $\mathbf{g}$ are the Green's functions of the coupled (SL/ cap layer) system and of the infinite SL, respectively. The trace in Eqs. (28)-(30) is taken over the components $x x$ and $z z$, which contribute to the sagittal modes we are studying in this paper. The integration over $z$ and the summation over $n$ can be performed very easily because the Green's functions are only composed of exponential terms. ${ }^{53}$ Let us notice that if the homogeneous medium 0 is semi-infinite instead of finite, we calculate $\Delta n_{0}\left(\omega^{2}\right)$ instead of $n_{0}\left(\omega^{2}\right)$, where the contribution of the infinite homogeneous medium is subtracted [more details about the calculation of these variational densities of states (VDOS) are given in Ref. 8]. 
TABLE I. Speed velocities of sound and mass densities of Plexiglas, aluminum, water, and mercury.

\begin{tabular}{cccl}
\hline \hline & $\rho\left(\mathrm{g} / \mathrm{cm}^{3}\right)$ & $v_{t}\left(10^{5} \mathrm{~cm} / \mathrm{s}\right)$ & $v_{\ell}(\mathrm{km} / \mathrm{s})$ \\
\hline Plexiglas & 1200 & 1.38 & 2.7 \\
Aluminum & 2700 & 3.15 & 6.45 \\
Water & 1000 & & 1.49 \\
Mercury & 13500 & & 1.45 \\
\hline \hline
\end{tabular}

\section{NUMERICAL RESULTS AND DISCUSSIONS}

We now illustrate these theoretical results by a few numerical calculations for some specific examples. We report the results of dispersion relations, densities of states of acoustic waves in semi-infinite solid-fluid SLs made of Plexiglas-water and aluminum-water with a free surface or capped with a mercury fluid medium of finite or infinite extent. The existence of allowed and forbidden bands in these structures has been shown theoretically ${ }^{45}$ and verified experimentally. ${ }^{48,49}$ The thicknesses of the fluid and solid layers are assumed to be equal, $d_{f}=d_{s}$, and the period $D$ $=d_{f}+d_{s}=2 d_{f}$ as in Refs. 45 and 48 . Table I gives the numerical values of speed velocities of sound and mass densities of the materials used in this work. We shall focus our attention first on the existence and behavior of acoustic waves associated with a free surface of the SL depending on whether the latter is terminated with a fluid layer or a solid layer. In particular, for a fluid layer termination, we shall generalize a rule obtained before on the existence of shear horizontal surface waves in solid-solid SLs, namely the creation of two semi-infinite SLs obtained by the cleavage of an infinite SL along a plane parallel to the interfaces inside the fluid layer gives rise to one mode per gap for any value of the wave vector $k_{\|}$. In contrast, for a solid termination, this rule is not fulfilled. In particular, zero, one, or two modes may appear in the gaps of two semi-infinite SLs. Then, we address the interface modes between a solid-fluid SL and a homogeneous fluid and show that this interface may support new interface modes in comparison with the interface between two homogeneous solid-fluid media. Finally, we investigate the problem of localized and resonant guided modes induced by a fluid of finite size (cap layer) made of mercury. In particular, we show that the band-gap structure of the SL can be used as a tool for confining the standing modes inside a fluid layer adsorbed on top of the SL.

\section{A. Semi-infinite superlattice in contact with vacuum}

Figure 2 gives the dispersion of bulk bands and surface modes, i.e., the reduced frequency $\Omega=\omega D / v_{t \text { (Plexiglas) }}$ as a function of the wave vector $k_{\|} D$ for two complementary semi-infinite Plexiglas-water SLs obtained by the cleavage of the infinite SL at the interface between a solid and a fluid layer. The gray areas are the bulk bands where acoustic waves are allowed to propagate in these structures. Theses areas are separated by forbidden bands (gaps). One can notice that because of the small contrast between Plexiglas and water acoustic parameters, the gaps are not very large as

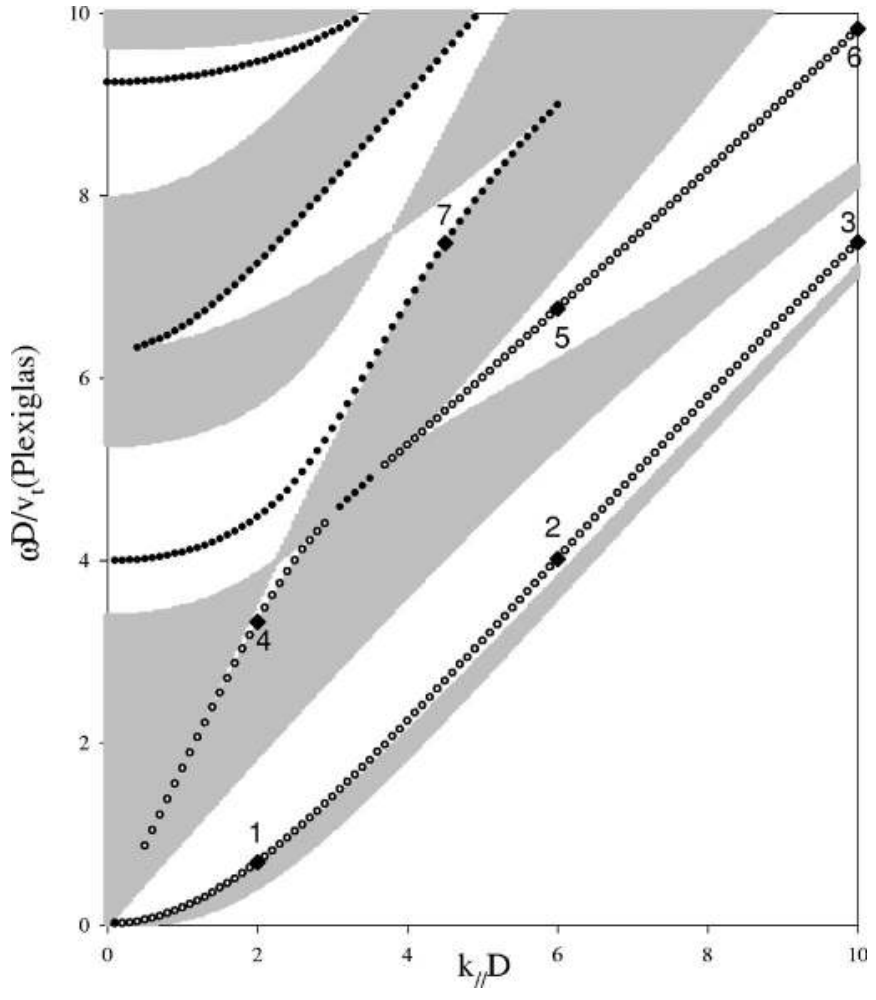

FIG. 2. Bulk and surface sagittal acoustic waves in a Plexiglas/ water SL. The curves give $\omega D / v_{t(\text { Plexiglas) }}$ as a function of $k_{\|} D$, where $\omega$ is the frequency, $k_{\|}$is the propagation vector parallel to the interface, $v_{t \text { (Plexiglas) }}$ is the transverse speed of sound in Plexiglas, and $D$ is the period of the SL. The gray areas represent the bulk bands. The dots represent the surface modes for the semi-infinite SL terminated by a water layer. The open circles represent the surface modes for the complementary SL terminated by a Plexiglas layer.

compared to Al-water SL (see below). The two lowest bands lying below the velocities of sound in both Plexiglas and water are constituted by evanescent waves in these two media. These two bands tend asymptotically to the interface mode between Plexiglas and water for large values of $k_{\|} D$. The creation of the surface of the SL gives rise to surface modes inside the gaps. The dots (open circles) represent surface modes when a water layer (Plexiglas layer) is at the surface of the SL. These modes are obtained from Eqs. (13), (14), (23), and (24). At $k_{\|} D=0$ (normal incidence), there is a decoupling between longitudinal and transverse waves in solid layers and the band-gap structure results only from the interaction between longitudinal waves in solid and fluid layers. In this case, it is known that the surface modes appear on the surface layer of the SL that has a lower acoustic impedance $^{23} Z=\rho v$. This is clearly shown in Fig. 2, where all the surface modes appear on the surface terminated by a water layer since $Z_{\text {water }}<Z_{\text {Plexiglas. }}$ By increasing $k_{\|} D$, these surface modes still exist in the highest gaps until the closing of the gaps. When the SL terminates with a Plexiglas layer, we obtain two branches (open circles). When $k_{\|} D$ increases, one of these branches (the lowest) tends to the interface mode between Plexiglas and water in the same way as the two lowest bands do. However, the highest branch tends asymptotically to the Rayleigh wave at the surface of the Plexi- 

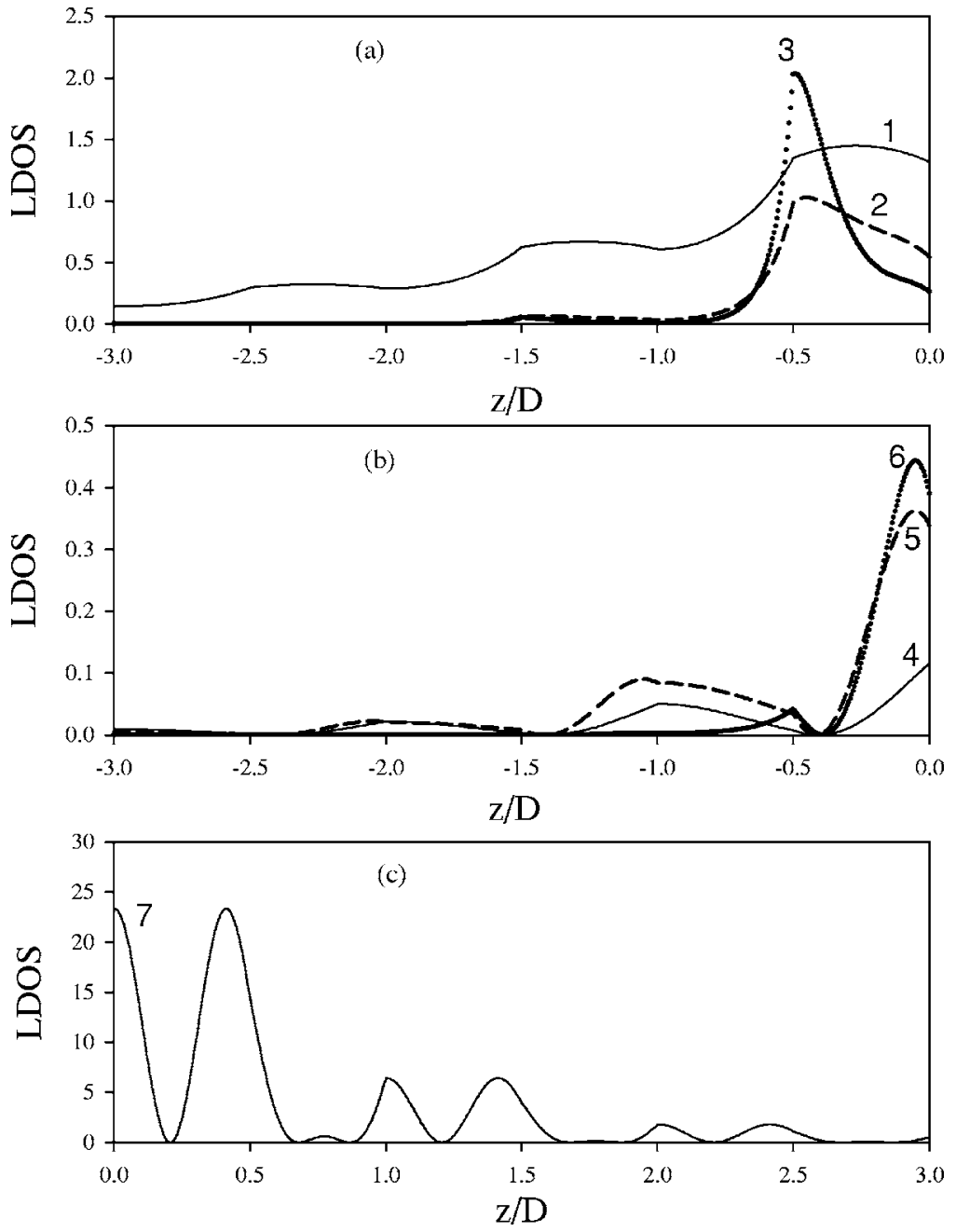

FIG. 3. Spatial representation of the local density of states (LDOS) corresponding to localized modes labeled 1,2,3 (a) and 4,5,6 (b) in Fig. 2 at $k_{\|} D=2,6$, and 10 , respectively. (c) The same as in (a) and (b) but for the localized mode labeled 7 in Fig. 2 at $k_{\|} D=4.5$. glas layer. This is clearly shown in the local density of states (LDOS) sketched in Figs. 3(a) and 3(b) for the modes labeled $1,2,3$ and 4,5,6 at $k_{\|} D=2,6$, and 10, respectively. The LDOS reflects the square modulus of the displacement field in the layers. One can see clearly [Fig. 3(a)] that the modes in the lowest branch (labeled 1, 2, and 3 in Fig. 2) become localized at the interface between Plexiglas and water when increasing $k_{\|} D$. However, the modes in the highest branch (labeled 4, 5, and 6 in Fig. 2) represent Rayleigh waves localized at the surface of the Plexiglas layer [Fig. 3(b)]. We have also drawn [Fig. 3(c)] the LDOS for the mode labeled 7 at $k_{\|} D=4.5$ in Fig. 2. This mode shows a strong localization at the surface of the water layer and decreases when penetrating into the SL.

An interesting result in Fig. 2 is the existence of one mode per gap associated with either one or the other of the two complementary semi-infinite SLs. The origin of this result comes from the analysis of the variational density of states (VDOS) $\Delta n_{s}(\omega)$ [respectively, $\left.\Delta n_{f}(\omega)\right]$ between the semiinfinite SL terminated by a solid layer (respectively, a fluid layer) and the same amount of the bulk SL as described in Sec. II C. These $\Delta n_{s}(\omega)$ and $\Delta n_{f}(\omega)$ are plotted in Figs. 4(a) and $4(\mathrm{~b})$ for $k_{\|} D=4.5$, as a function of the reduced frequency $\Omega$. The $\delta$ functions appearing at the bulk band edges and at the frequencies of the surface modes are enlarged by adding a small imaginary part to the frequency $\omega$. The $\delta$ functions associated with the surface localized modes are noted $L_{i}$, and the $\delta$ functions of weight $(-1 / 4)$ situated, respectively, at the bottom and top of any bulk band $i$ are called $B_{i}$ and $T_{i}$. The form of these latter enlarged $\delta$ functions $B_{i}$ and $T_{i}$ is not exactly the same because of the contributions coming from the divergence in $\left(\omega-\omega_{B_{i}}\right)^{-1 / 2}$ or $\left(\omega-\omega_{T_{i}}\right)^{-1 / 2}\left(\omega_{B_{i}}\right.$ and $\omega_{T_{i}}$ are the frequencies of the bottom and the top of every bulk band of the SL), existing near the band edges in the densities of states in 1D systems. Apart from the above $\delta$ peaks and the particular behavior near the band edges, the VDOS does not show any other significant effect inside the bulk bands of the SL. Now, by considering the variational DOS $\Delta n(\omega)$ $=\Delta n_{s}(\omega)+\Delta n_{f}(\omega)$ between the two complementary semiinfinite SLs and the initial infinite SL, given in Figs. 4(a) and 4(b), one can show both analytically and numerically [Fig. $4(\mathrm{c})]$ that (i) $\Delta n(\omega)$ is equal to zero for $\omega$ falling inside any SL bulk band, and (ii) the loss of modes due to the peaks of weight $(-1 / 2)$ at every edge of the bulk bands (as we consider two semi-infinite SLs) is then compensated by the gain associated with the localized states $\left(L_{1}, L_{2}, L_{3}, L_{4}\right)$ inside the gaps in order to ensure the conservation of the total number of states.

A remarkable point to notice is the generalization of this rule when the cleavage is produced inside a fluid layer along 

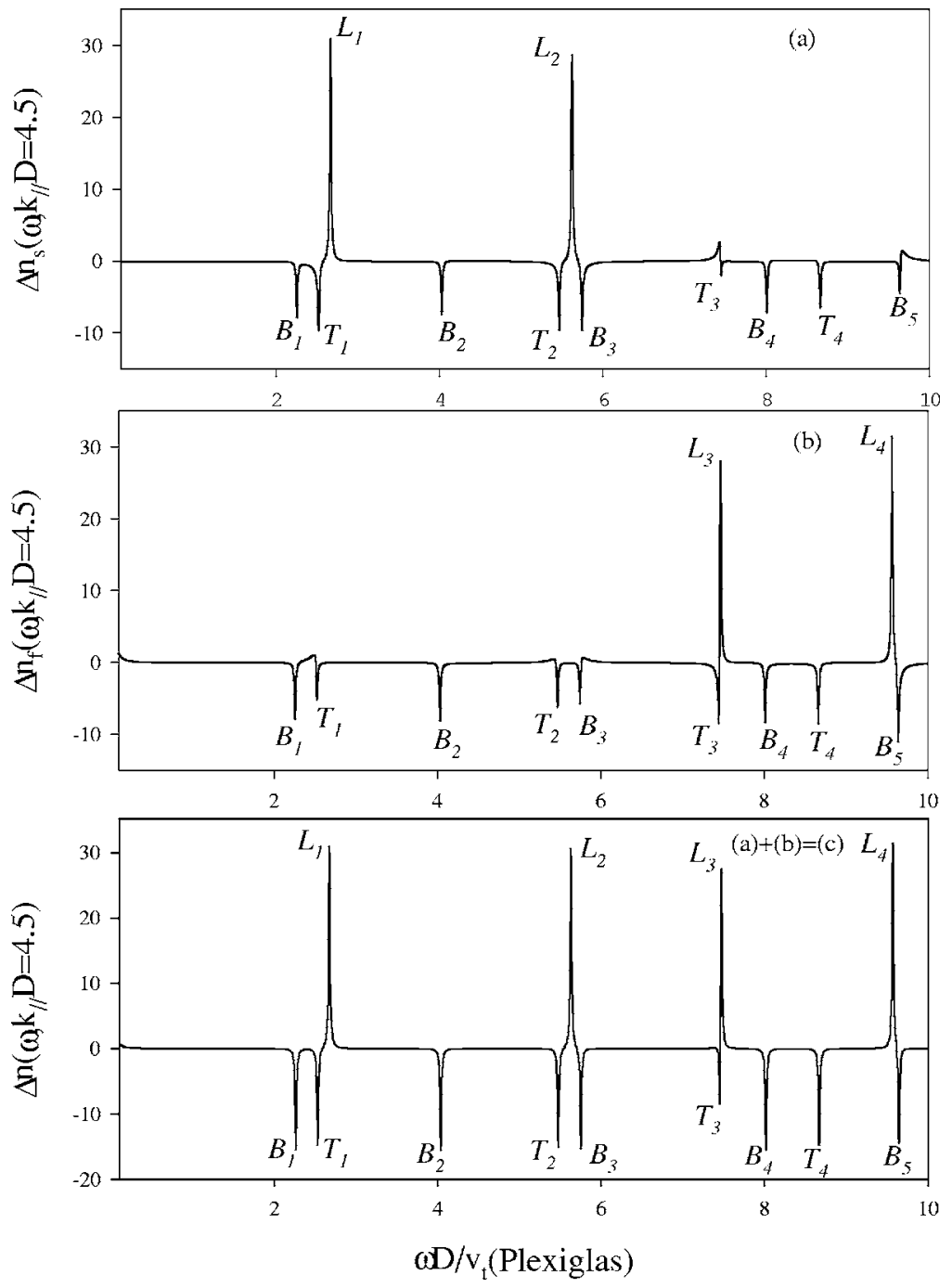

FIG. 4. (a) The variation of the density of states (VDOS) of sagittal waves [in units of $\left.D / v_{t \text { (Plexiglas) }}\right]$ between a semi-infinite SL terminated by a complete solid layer and the same amount of the infinite SL, as a function of $\omega D / v_{t \text { (Plexiglas) }}$ at $k_{\|} D=4.5 . B_{i}$ and $T_{i}$, respectively, refer to $\delta$ peaks of weight $(-1 / 4)$ situated at the bottom and the top of the bulk bands and $L_{i}$ indicates the localized surface modes. (b) The same as in (a) but for the complementary SL terminated by a complete fluid layer. (c) The same as in (a) and (b), but for two complementary SLs (a) and (b). a plane parallel to the interfaces [Fig. 5(a)]. These results are obtained from Eqs. (10) and (11) by taking the fluid layer of the same nature as the bulk fluid (water) but with different thickness: $d_{f 1}=0.6 d_{f}$ open circles and $d_{f 2}=0.4 d_{f}$ dots. The results in Fig. 5(a) clearly show the existence of one mode per gap and the dependence of the surface modes on the thickness of the water layer at the surface. To give a better insight into this general result, we present in Fig. 5(b) the variation of the surface modes as a function of the widths $d_{f 1}$ and $d_{f 2}$ of the surface fluid layers for two complementary SLs such that $d_{f 1}+d_{f 2}=d_{f}$ at $k_{\|} D=4.5$. The dots (open circles) are surface modes induced by the water layer of thickness $d_{f 1}\left(d_{f 2}\right)$. One can see clearly that for any combination of two complementary SLs such that $d_{f 1}+d_{f 2}=d_{f}$, there is usually one surface state per gap. This is valid for any value of the wave vector $k_{\|} D$. However, a very specific case in which no surface modes appear inside the gaps occurs when the cleavage is produced exactly at the middle of the fluid layer, i.e., $d_{f 1}=d_{f 2}=0.5 d_{f}$.

In order to show the dependence of the band-gap structure as well as the surface modes on the nature of the solid constituting the SL, we have plotted in Fig. 6 the reduced frequency $\Omega=\omega D / v_{t(\mathrm{Al})}$ as a function of the reduced parallel wave vector $k_{\|} D$ for a SL composed of $\mathrm{Al}$ and water layers with $d_{f}=d_{s}=D / 2$. Due to the large acoustic contrast between these two materials, we obtain large gaps in comparison with the case of Plexiglas-water SL. Also, the dispersion of the surface modes exhibits quite different behavior. For example, when the cleavage is carried out between an $\mathrm{Al}$ and a water layer of the infinite SL [Fig. 6(a)], one observes that apart from the lowest branch (open circles) lying at the surface of the Al layer, all the other branches (dots) are localized at the surface of the water layer. Figure 6(b) gives the same results as in Fig. 6(a) but for two complementary SLs terminated with two fluid layers such that $d_{f 1}=0.4 d_{f}$ (dots) and $d_{f 2}$ $=0.6 d_{f}$ (open circles). These results show again the existence of one mode per gap and the dependence of the surface modes on the thickness of the fluid layer at the surface.

Now we turn to the case in which the cleavage occurs inside a solid layer along a plane parallel to the interfaces. Figures 7(a) and 7(b) show the dispersion curves for two semi-infinite complementary Al-water SLs ending with incomplete Al surface layers such that $d_{s 1}=d_{s 2}=d_{s} / 2[$ Fig. $7(\mathrm{a})]$ and $d_{s 1}=0.2 d_{s}, d_{s 2}=0.8 d_{s}[$ Fig. $7(\mathrm{~b})]$. These results are obtained from Eqs. (15) and (16) by considering the solid cap layer of the same nature as those constituting the bulk SL but with different thicknesses. In contrast to the case we cut in the middle of a fluid layer where no surface modes exist, 

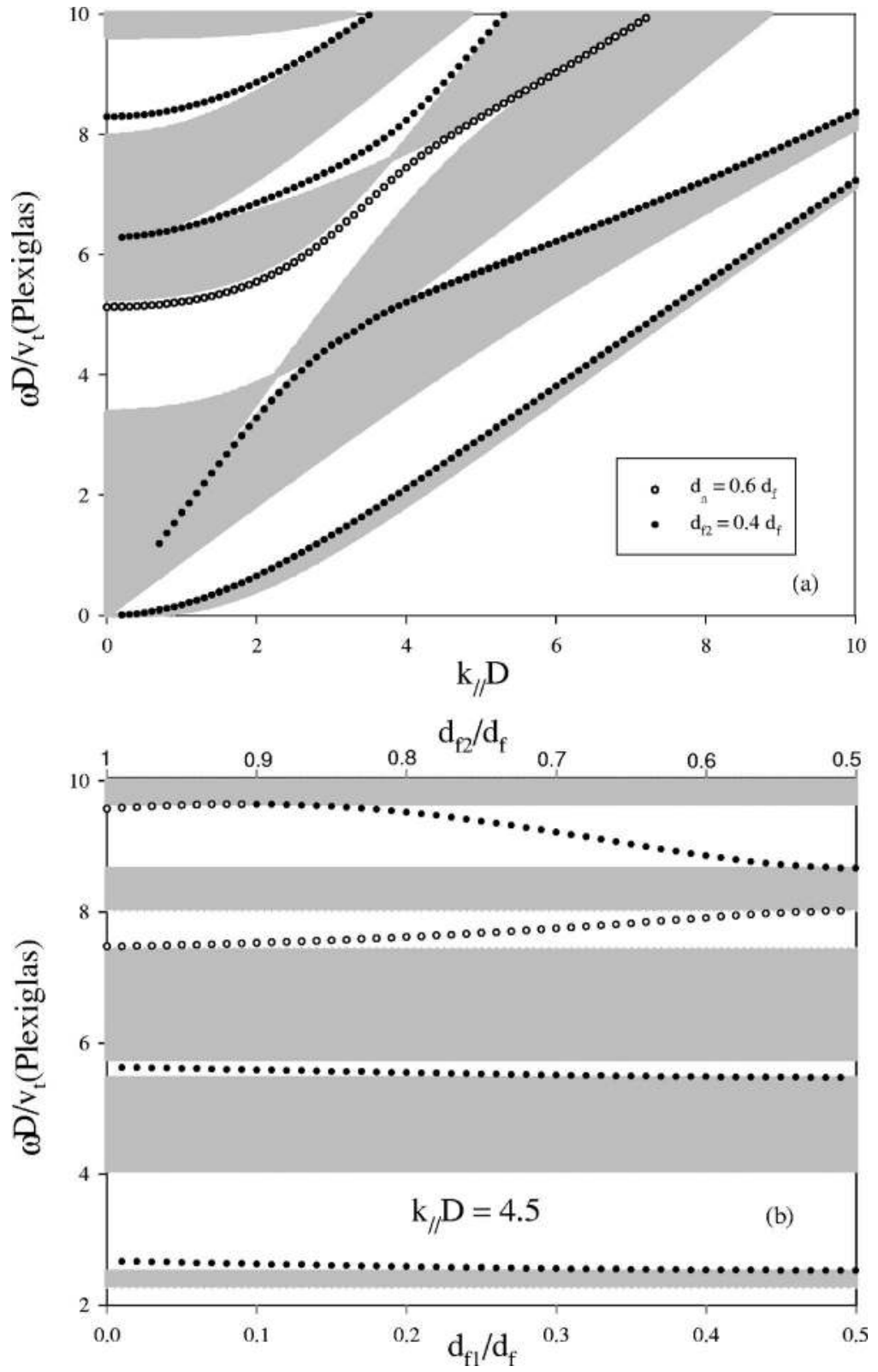

FIG. 5. (a) The same as in Fig. 2, but for two complementary SLs obtained by the cleavage of an infinite SL inside a fluid layer such that $d_{f 1}$ $=0.4 d_{f}$ (dots) and $d_{f 2}=0.6 d_{f}$ (open circles). (b) Dimensionless frequencies $\left(\omega D / v_{t \text { (Plexiglas) }}\right)$ of the localized modes induced by a water cap layer of thickness $d_{f 1}$ (dots) and $d_{f 2}$ (open circles) for $k_{\|} D=4.5 . d_{f 1}$ and $d_{f 2}$ are chosen such that $d_{f 1}$ $+d_{f 2}=d_{f}$.
Fig. 7(a) shows that cutting in the middle of a solid layer induces two degenerate surface branches in some gaps and no surface modes in other gaps for two complementary SLs. Starting from this situation, one can notice [Fig. 7(b)] a lifting of the degeneracy of the surface modes as far as $d_{s 1}$ and $d_{s 2}$ become different from $0.5 d_{s}$. Therefore, zero, one, or two surface branches may exist inside the different gaps in the $\left(\Omega, k_{\|} D\right)$ plane. This is clearly shown in Fig. 8, where we have plotted the evolution of the surface modes for two complementary SLs with different thicknesses $d_{s 1}$ and $d_{s 2}$ such that $d_{s 1}+d_{s 2}=d_{s}$ at $k_{\|} D=7$. One can notice that (i) for $d_{s 1}=0$ and $d_{s 2}=d_{s}$, there is one mode per gap (as discussed before), (ii) for $d_{s 1}=d_{s 2}=0.5 d_{s}$, there are two degenerate branches in the lowest three gaps and no modes in the highest three gaps, and (iii) for arbitrary values of $d_{s 1}$ and $d_{s 2}$ (with $d_{s 1}+d_{s 2}=d_{s}$ ), there may exist zero, one, or two modes for any combination of the two complementary SLs. The results for two Plexiglas-water SLs with complementary solid layer thicknesses at the surface are sketched in Fig. 9. These results show the same conclusions as in Fig. 7, namely the existence of two degenerate branches in some gaps and no modes in other gaps for $d_{s 1}=d_{s 2}=0.5 d_{s}$ [Fig. 9(a)], whereas far from this situation there may exist zero, one, or two modes in each gap for two complementary SLs [Fig. 9(b)].

\section{B. Semi-infinite superlattice in contact with a fluid}

In this section, we study localized and resonant modes induced by the interface between a semi-infinite SL in contact with a semi-infinite homogeneous fluid or a semi-infinite SL capped with a finite fluid cap layer.

First, we consider the interface modes induced by the interface between a semi-infinite Plexiglas-water SL terminated by a Plexiglas layer and a semi-infinite fluid made of mercury (Hg). Figure 10(a) gives the dispersion of localized 

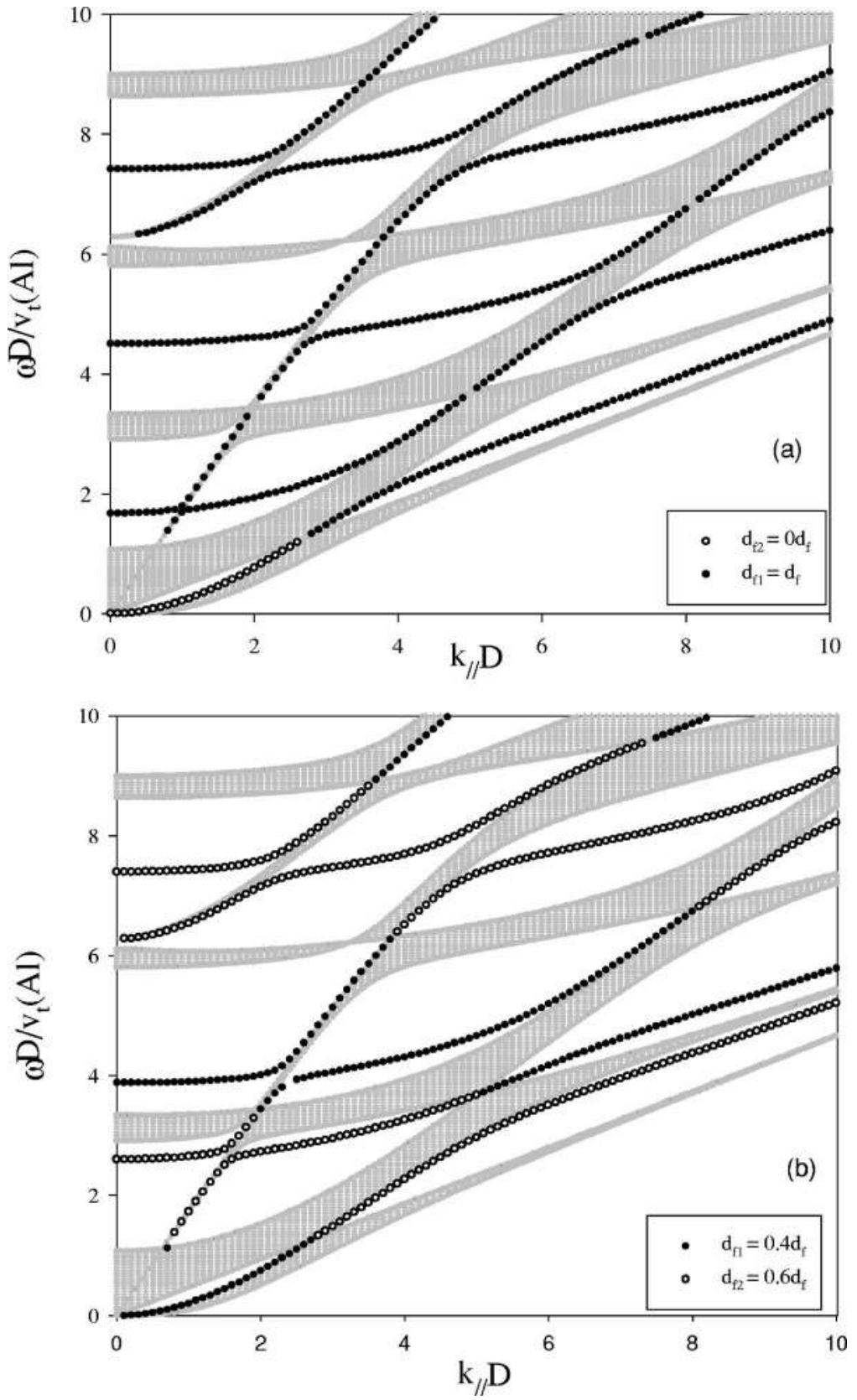

FIG. 6. (a) The same as in Fig. 2 but for the Al-water SL. The curves give the dimensionless frequency $\omega D / v_{t(\mathrm{Al})}$ as a function of the reduced wave vector $k_{\|} D$. (b) The same as in Fig. 5(a) but for the Al-water SL. (resonant) modes induced by this interface below (above) the $\mathrm{Hg}$ bulk band (straight line). These modes are obtained from the maxima of the variation of the DOS $\Delta n(\omega)$ between the coupled SL-Hg system and the same amount of the bulk SL and of the bulk $\mathrm{Hg}$ (as described in Sec. II C). Two examples of $\Delta n(\omega)$ are plotted in Figs. 10(b) and 10(c) for $k_{\|} D=2.5$ and 7 , respectively. The $\delta$ functions of weight $(-1 / 4)$ situated, respectively, at the bottom and top of any bulk band are called $B_{i}$ and $T_{i} . B_{f}$ refers to a $\delta$ peak of weight $(-1 / 4)$ situated at the bottom of the $\mathrm{Hg}$ fluid bulk band. The two positive $\delta$ functions lying below $B_{f}$ are true localized interface modes; their positions may be deduced also from Eqs. (15) and (16). However, the small peaks lying above $B_{f}$ are interface resonances [labeled $R_{1}$ and $R_{2}$ in Fig. 10(b)]. The latter modes are evanescent when penetrating into the SL and propagate in the $\mathrm{Hg}$ medium as it is shown in the LDOS sketched in the inset of Fig. 10(b) for the resonance $R_{2}$. Let us recall that the interface localized modes in Fig. 10(b) are enlarged artificially by adding a small imaginary part to the frequency $\omega$, whereas the interface resonances are intrinsically widened because of their interaction with the $\mathrm{Hg}$ bulk band. Resonances $R_{1}$ and $R_{2}$ could be detected experimentally from the reflection coefficient of an incident wave launched in the $\mathrm{Hg}$ homogeneous medium. The two localized branches lying below the $\mathrm{Hg}$ bulk band exhibit different spatial localization. The lowest branch shows a strong localization at the interface $\mathrm{SL} / \mathrm{Hg}$, whereas the highest branch shows an important localization at the internal interface between Plexiglas and water just below the $\mathrm{SL} / \mathrm{Hg}$ interface. These results are illustrated in the LDOS sketched in the inset of Fig. 10(c) by dashed and solid curves associated with the lowest and highest modes labeled, respectively, $L_{3}$ and $L_{4}$ 

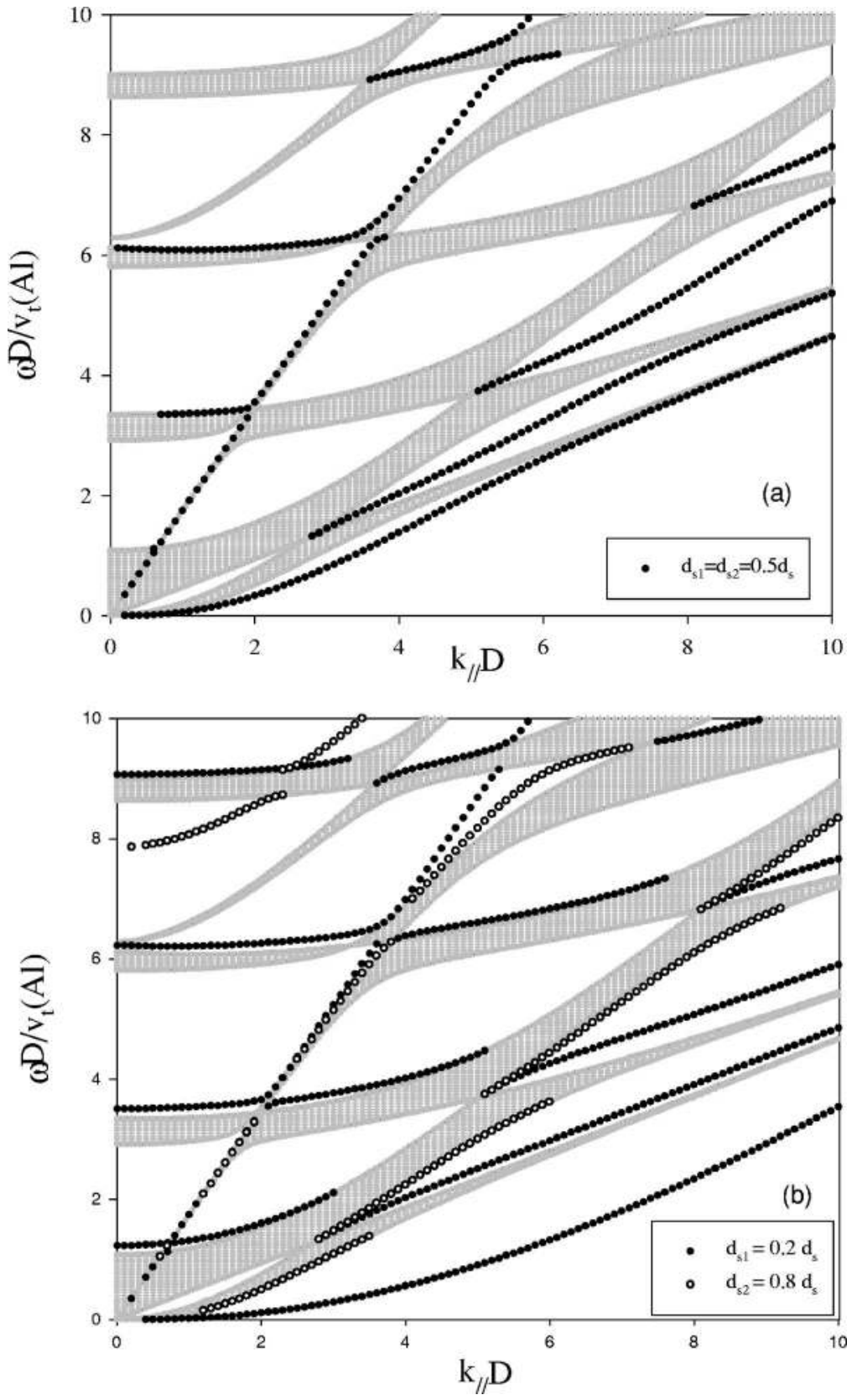

FIG. 7. (a),(b) The same as in Fig. 2 but for two complementary SLs obtained by the cleavage of an infinite SL inside a solid layer such that (a) $d_{s 1}=d_{s 2}=0.5 d_{s}$ and (b) $d_{s 1}=0.2 d_{s}$ and $d_{s 2}=0.8 d_{s}$. in Fig. 10(c). Now, if the $\mathrm{Hg}$ medium is in contact with an Al-water SL (not given here), one obtains only one interface branch below the $\mathrm{Hg}$ sound line, which is mainly localized at the $\mathrm{SL} / \mathrm{Hg}$ interface. Besides this interface localized branch, one obtains one resonant branch above the $\mathrm{Hg}$ sound line. Let us mention that the interface between a finite-size SL and a homogeneous liquid can be used to enhance the resonant transmission of acoustic waves from a SL into a liquid. ${ }^{55}$

Now we assume that the mercury medium is of finite size (with a thickness $d_{0}$ ) instead of being semi-infinite in extent. Figures 11(a) and 11(b) give the dispersion of localized and resonant modes (open circles) induced by a cap layer of width $d_{0}=0.5 \mathrm{D}$ and $1.5 \mathrm{D}$, respectively. The straight line indicates the sound line in $\mathrm{Hg}$. These modes are obtained as well-defined peaks (not shown here) in the variation of the DOS $\Delta n(\omega)$ between the capped SL and the same amount of the bulk SL without the cap layer (see Sec. II C). The local- ized modes inside the gaps can be obtained from the dispersion relations [Eqs. (10) and (11)].

The localized and resonant modes induced by the cap layer can be divided into three categories according to the behavior of the corresponding eigenstates along the axis of the SL; they may propagate in both the SL and the cap layer (pseudoguided waves) when their frequencies fall inside the SL bulk band and above the $\mathrm{Hg}$ sound line, or propagate in the cap layer and decay in the SL (guided waves) when their frequencies fall inside the SL gaps and above the $\mathrm{Hg}$ sound line, or decay on both sides of the SL-Hg interface when their frequencies lie inside the SL gaps and below the $\mathrm{Hg}$ sound line. In Fig. 11(a), one can notice that for a small thickness of $\mathrm{Hg}\left(d_{0}=0.5 \mathrm{D}\right)$, all the guided modes are localized within the gaps of the SL, whereas for a large value of $d_{0}\left[d_{0}=1.5 D\right.$, Fig. $\left.11(\mathrm{~b})\right]$, the localized modes lying inside the gaps continue to exist as well-defined resonances (or 


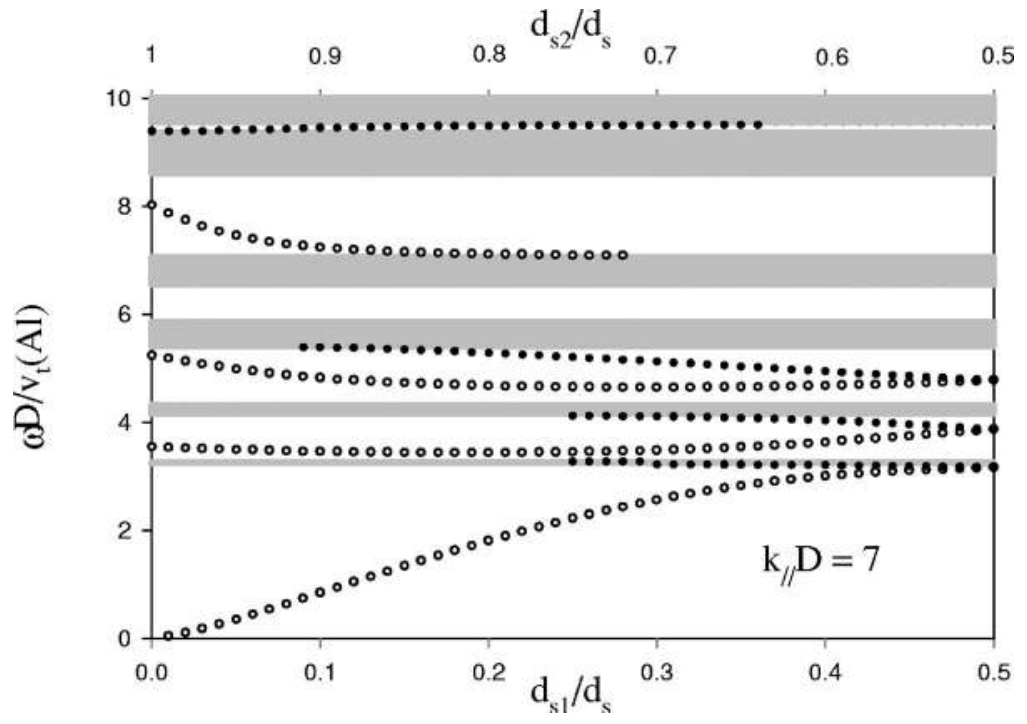

FIG. 8. Dimensionless frequencies $\left(\omega D / v_{t(\mathrm{Al})}\right)$ of the localized modes induced by a solid cap layer of thickness $d_{s 1}$ (dots) and $d_{s 2}$ (open circles) for $k_{\|} D=7 . d_{s 1}$ and $d_{s 2}$ are chosen such that $d_{s 1}+d_{s 2}=d_{s}$.

leaky waves) inside the SL bulk bands. The two lowest branches lying below the $\mathrm{Hg}$ sound line in Figs. 11(a) and 11(b) coincide with the interface localized branches in Fig. $10(\mathrm{a})$.

These results show that the frequencies of the localized and resonant modes are very dependent upon the thickness $d_{0}$ of the cap layer. A better insight into this variation is shown in Fig. 12(a) for $k_{\|} D=3$. The lowest two branches that correspond to localized modes at the cap layer-SL interface become almost independent of $d_{0}$ for $d_{0} \geqslant 0.5 D$ [see also Figs. 11(a) and 11(b)]. The higher branches corresponding to the $\mathrm{Hg}$ guided modes become close to each other when $d_{0}$ increases. Let us also notice that the curves in this figure become almost flat when a localized branch is going to become resonant by merging into a bulk band, whereas the variation with $d_{0}$ becomes faster when the resonant branch penetrates deep into the band. The intensity of the resonant modes in the DOS (not shown here) decreases or even vanishes when $d_{0}$ is small or the frequency is high. Finally, let us mention that for any given frequency in Fig. 12(a), there is a periodic repetition of the modes as a function of $d_{0}$.

Figure 12(b) gives a better insight into the variation of the two lowest interface branches as a function of $d_{0}$ for $k_{\|} D$ $=7$. For $d_{0}=0$, the two branches coincide with the surface modes of the SL terminated with a Plexiglas layer (Fig. 2). When $d_{0}$ increases, these two branches decrease, cross the lowest bands, and tend asymptotically to the localized branches associated to the SL/Hg interface [Fig. 10(a)]. The spatial localization of these two branches depends strongly on $d_{0}$. Indeed the highest branch, first strongly localized at the surface of the SL for $d_{0}=0$ [Fig. 3(b)], becomes localized at the internal surface between Plexiglas and water for $d_{0}$ $\geqslant 0.5 D$ [see the solid curve in the inset of Fig. 10(c)]. In contrary, the lowest branch, first strongly localized at the internal surface between Plexiglas and water for $d_{0}=0$ [Fig. $3(\mathrm{a})$, becomes localized at the interface $\mathrm{SL} / \mathrm{Hg}$ when $d_{0}$ $\geqslant 0.5 D$ [see the dashed curve in the inset of Fig. 10(c)].

It is worthwhile to notice that the detection of surface acoustic waves in solid-fluid SL with a fluid cap layer can be achieved by means of reflection coefficient measurements. ${ }^{56}$ Indeed, an incident wave launched from a semi-infinite sub-
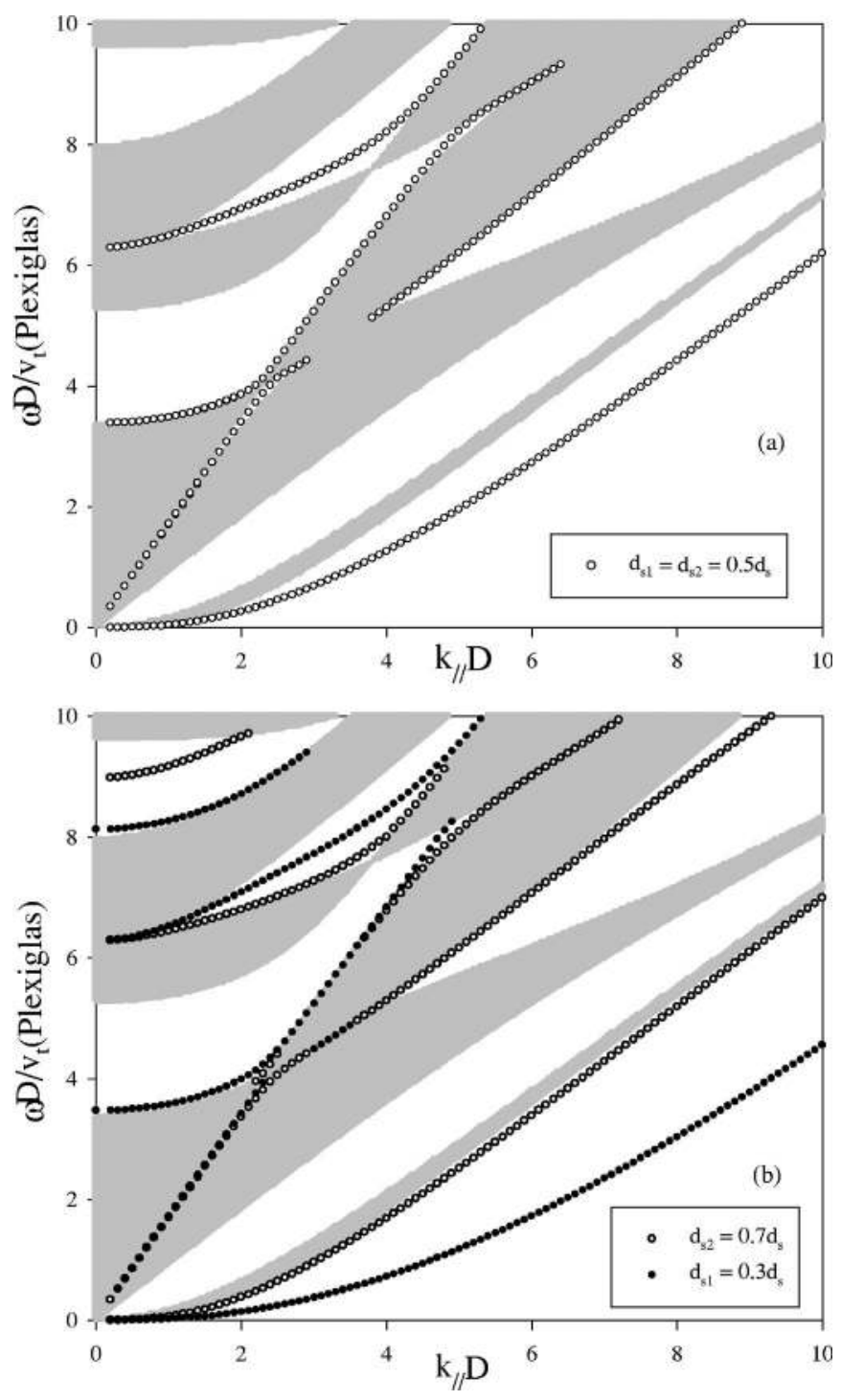

FIG. 9. The same as in Fig. 2 but for two complementary Plexiglas-water SLs ended with a Plexiglas cap layer such that (a) $d_{s 1}=d_{s 2}=0.5 d_{s}$ and (b) $d_{s 1}=0.3 d_{s}$ and $d_{s 2}=0.7 d_{s}$. 

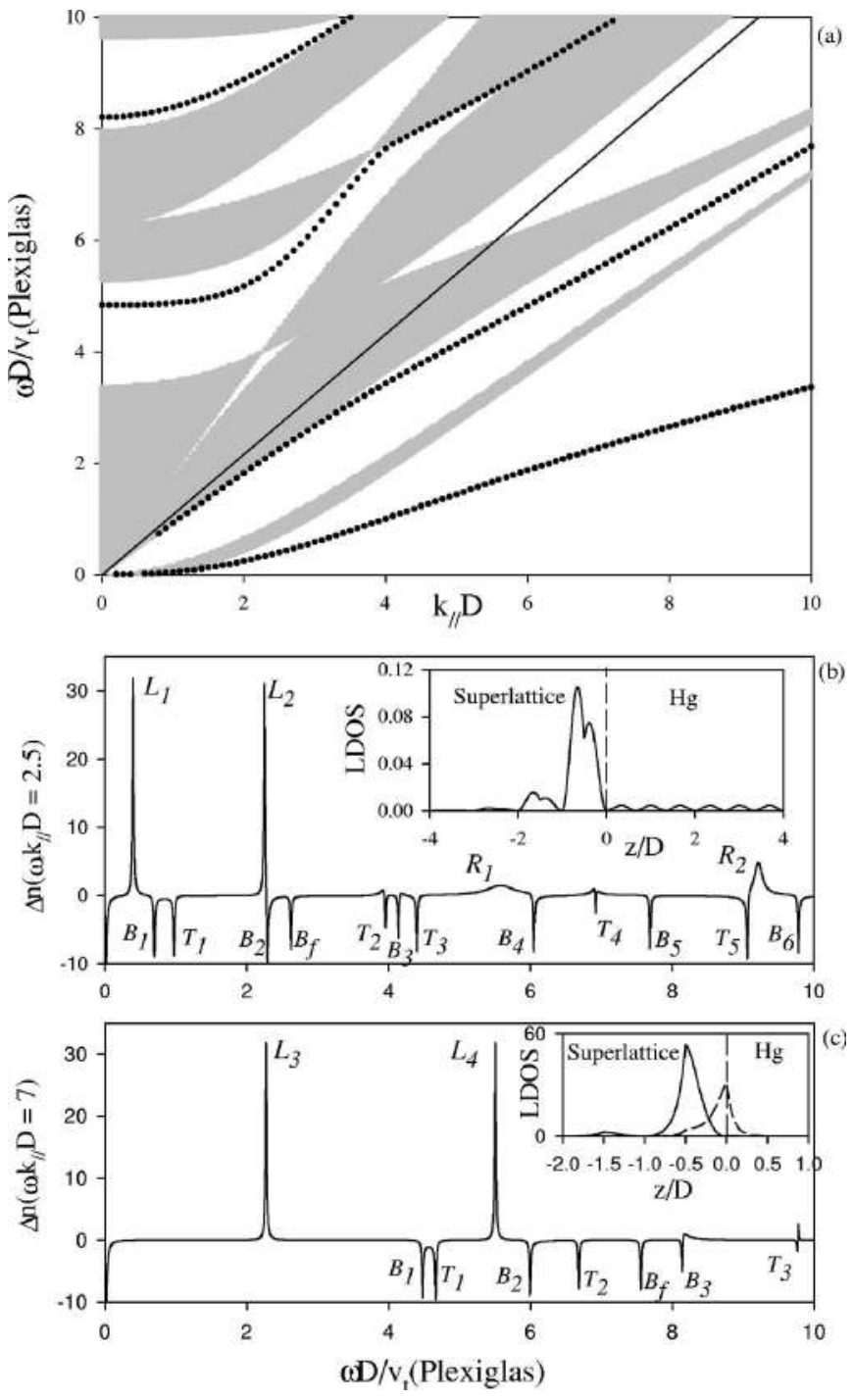

FIG. 10. (a) Dispersion of interface sagittal modes induced by the interface between a semi-infinite Plexiglas-water SL and a homogeneous fluid mercury $(\mathrm{Hg})$. The curves are localized (resonant) when their frequencies lie below (above) the velocity of sound in $\mathrm{Hg}$ indicated by a straight line. (b) The variation of the DOS of sagittal waves (in units of $D / v_{t(\text { Plexiglas })}$ ) between a semi-infinite SL in contact with $\mathrm{Hg}$ and the same amount of the infinite SL and the infinite $\mathrm{Hg}$, as a function of $\omega D / v_{t \text { (Plexiglas) }}$ at $k_{\|} D=2.5 . B_{i}$ and $T_{i}$, respectively, refer to $\delta$ peaks of weight $(-1 / 4)$ situated at the bottom and the top of the SL bulk bands, and $B_{f}$ refers to the bottom of the $\mathrm{Hg}$ bulk band. $L_{i}$ indicates localized interface modes, whereas $R_{i}$ indicates resonant modes. The inset gives the LDOS for the mode labeled $R_{2}$ in Fig. 10(b). (c) The same as in (b) but for $k_{\|} D$ $=7$. The dashed and full curves in the inset give the LDOS for the modes labeled, respectively, $L_{3}$ and $L_{4}$ in Fig. 10(c).

strate on top of which we deposit a finite SL with a fluid cap layer will be totally reflected back. Therefore, the amplitude of the reflected wave is unity and only its phase or equivalently the phase time, defined as the derivative of the phase with respect to the frequency $\omega$, may give information on the surface modes induced by the cap layer. Indeed, the surface modes appear as well defined peaks in the phase time, which is equivalent to the density of states (see Refs. 25, 26, and 57
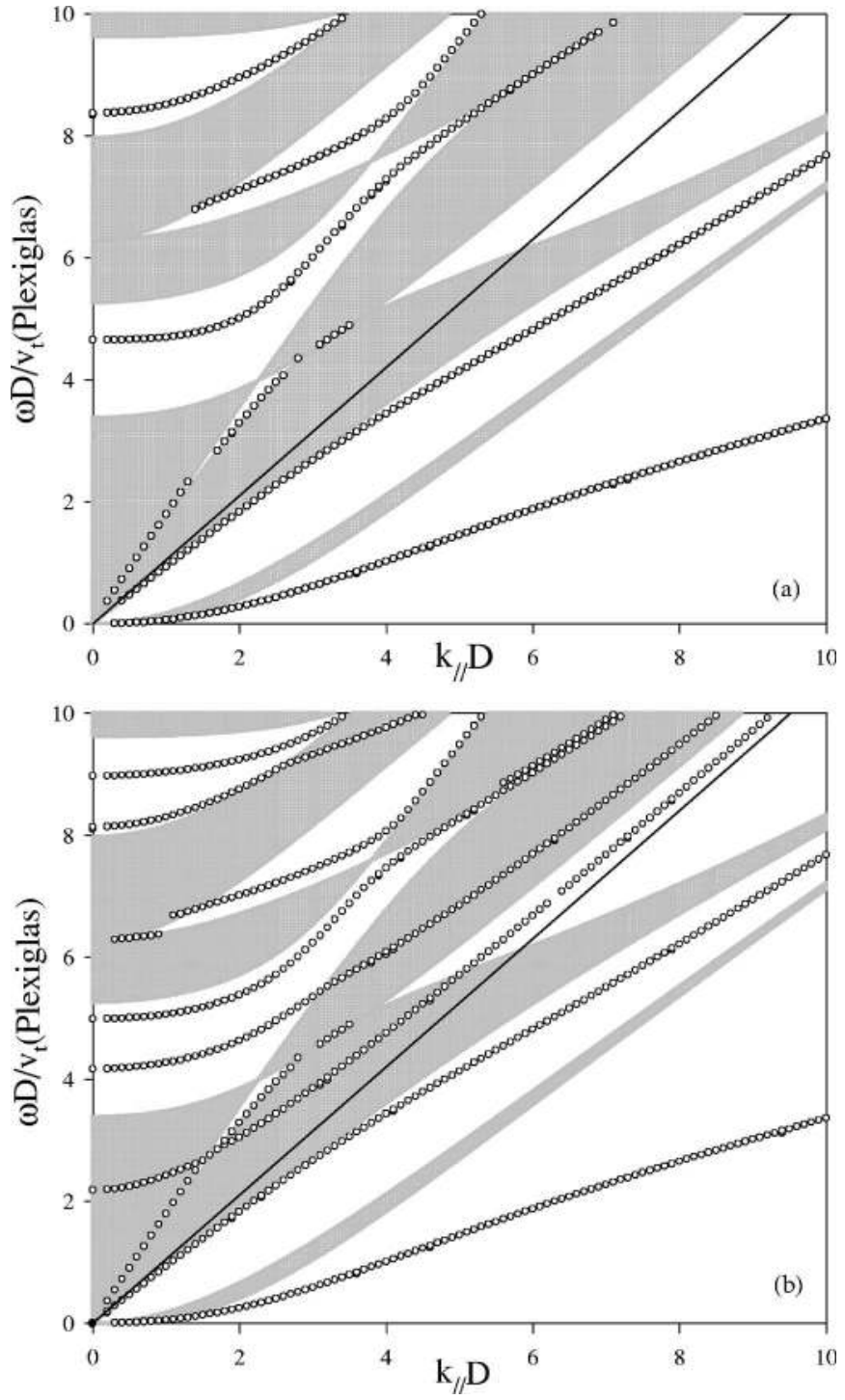

FIG. 11. (a) Dispersion of localized and resonant guided modes (open circles) induced by an adsorbed fluid layer of thickness $d_{0}$ $=0.5 \mathrm{D}$ deposited on top of the Plexiglas-water SL terminated by a Plexiglas layer. (b) The same as in (a) but for $d_{0}=1.5 \mathrm{D}$.

for more detail). Another method that enables us to deduce the guided modes of the fluid cap layer consists of putting a solid reflector on its top. The different modes of the structure are obtained from the minima of the reflection amplitudes. This method is reported in Ref. 58, where vibrations of an Al plate loaded with a water layer are demonstrated.

Another important quantity that may affect considerably the spectra of the density of states, reflection and transmission coefficients, and the corresponding phase times is the viscosity $\eta$ of the fluid. This quantity may be introduced by adding a small imaginary part to the square velocity in the fluid. ${ }^{50,51}$ Indeed, the viscosity may enlarge the $\delta$ peaks of the density of states in the same way as the artificial imaginary part added to the frequency $\omega$ does (see Fig. 4). Also, it was shown that the viscosity may reduce the intensity of the peaks in the reflection ${ }^{59}$ and transmission ${ }^{50,51}$ spectra. However, as mentioned before, if the fluid layer thickness is 

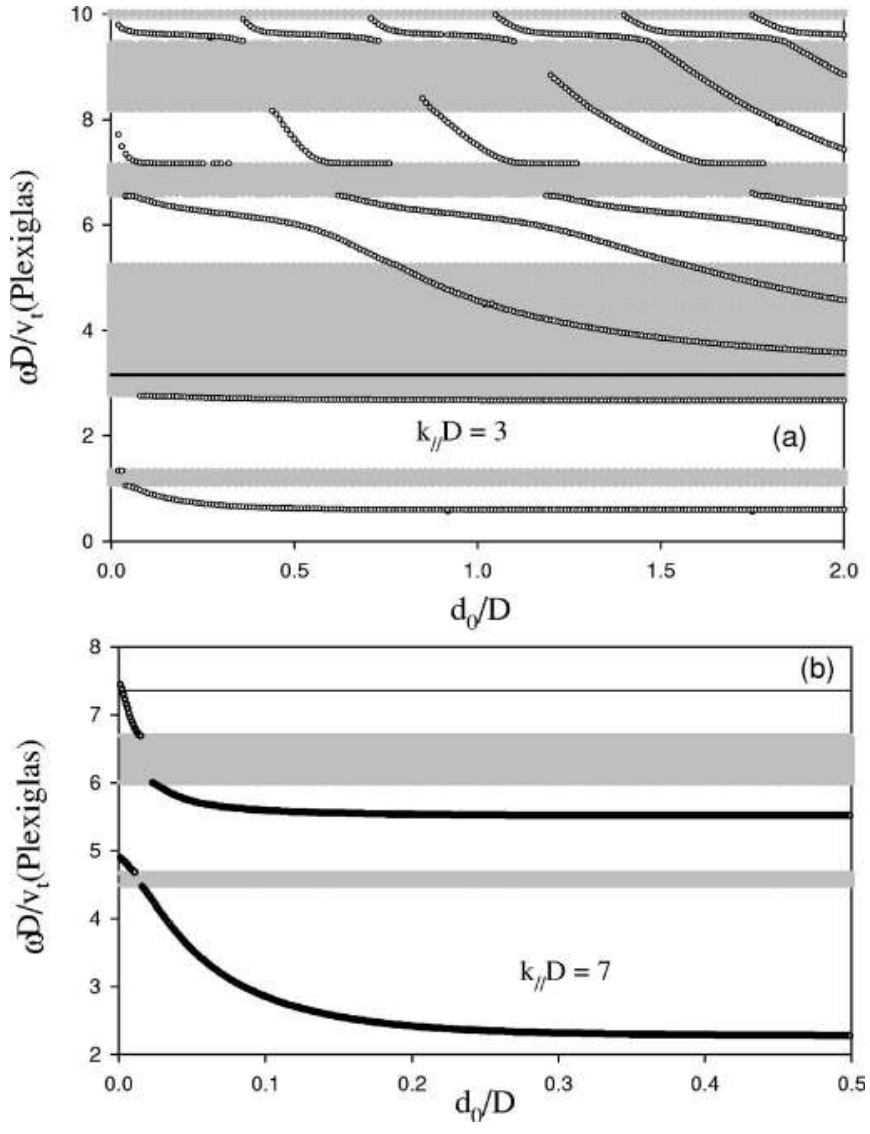

FIG. 12. (a) Frequencies of the localized and resonant modes induced by a $\mathrm{Hg}$ cap layer vs the width $d_{0}$ of the cap layer. The SL is the same as in Fig. 11 and $k_{\|} D=3$. The guided modes tend asymptotically to the sound velocity in $\mathrm{Hg}$ (indicated by a horizontal solid line) when $d_{0}$ increases, whereas the lowest two branches correspond to modes localized at the SL-cap layer interface. (b) The same as in (a) but for $k_{\|} D=7$.

greater than the viscous skin depth $\sigma=(2 \eta / \varrho \omega)$, then the assumption of an ideal fluid remains valid.

\section{CONCLUSIONS}

In this paper, we have presented an analytical calculation of the response function (Green's function) for acoustic waves of sagittal polarization in a semi-infinite SL made of alternating solid and fluid layers, with or without a cap layer or in contact with a homogeneous medium. These calculations enable us to deduce the local and total densities of states as well as the dispersion relations. These latter quantities are obtained in closed form that can be used by any reader interested in the subject without going into the details of the calculations. Although our results are obtained for solid-fluid SLs, they remain also valid for fluid-fluid SLs at oblique incidence. It is enough to take the transverse speed of sound in solid layers equal to zero. Of course, in practice, the two fluids would be separated by means of some latex material. The mass density and speed of sound in rubber are comparable to those of water. ${ }^{60}$ Hence, for a sufficiently thin latex partition, the presence of this extra layer should not affect the calculation in a significant way.
Different surface modes are obtained depending on whether the SL is terminated by a fluid layer or a solid layer. In the case of a fluid layer termination, we have generalized a rule about the existence of shear horizontal surface modes in solid-solid SLs, namely in creating two complementary semi-infinite SLs from cutting an infinite SL within a fluid layer, one obtains as many localized surface states as gaps for any value of $k_{\|}$. This result is based on the general rule about the conservation of the number of states and expresses a compensation between the loss of $1 / 2$ state at every bulk band edge (due to the creation of two free surfaces) and the gain due to the occurrence of surface states. However, the results are at variance if the cleavage is carried out inside a solid layer; in particular, the compensation of the loss of $1 / 2$ state at every edge of the bulk bands can be made by the existence of zero, one, or even two surface states in each gap.

We have also investigated the modes induced by a fluid cap layer at the surface of the SL and discussed the resulting guided and pseudoguided modes. When the cap layer is of semi-infinite extent, we obtain the interface modes between a SL and a homogeneous fluid. Here also, we have shown the existence of different interface and pseudointerface modes that are without analogue in the case of homogeneous media.

Let us finally mention that, similarly to the case of solidsolid SL, the solid-fluid layered media also present the property of Bragg acoustic mirrors and acoustic filters. ${ }^{40}$ In addition, these structures may present some particular features such as transmission zeros, Fano resonances, ${ }^{61}$ and Brewster acoustic angles ${ }^{62}$ that are without analogue in solid-solid SLs. These results together with an analytical calculation of the transmission and reflection coefficients in solid-fluid SLs will be presented in a forthcoming paper.

\section{ACKNOWLEDGMENTS}

Y.El H. and E.H. El B. gratefully acknowledge the hospitality of the Institut d'Electronique, de Microélectronique et de Nanotechnologie (IEMN), UMR CNRS 8520 and UFR de Physique, Université des Sciences et Technologies de Lille. This work was supported by "le Fond Européan de Développement Régional" (FEDER), and "le Conseil Régional Nord-Pas de Calais."

\section{APPENDIX}

The calculation of the Green's functions of an infinite SL (Fig. 1), a semi-infinite SL capped with a fluid layer at the surface [Fig. 1(b)], or a solid layer at the surface [Fig. 1(c)] requires [Eq. (1)] knowledge of the bulk Green's functions of an infinite fluid, an infinite solid, as well as the Green's functions of the corresponding layers in their space of interfaces $M$. Following the interface response theory, ${ }^{8,52}$ we obtained the following.

\section{Green's functions of infinite ideal fluid and an isotropic solid media}

The bulk Green's functions of an ideal fluid and an isotropic solid are given, respectively, by ${ }^{53,54}$ 


$$
G_{f}\left(x_{3}, x_{3}^{\prime}\right)=\left(\begin{array}{cc}
2 \delta\left(x_{3}-x_{3}^{\prime}\right)-\frac{k_{\|}^{2}}{\alpha_{f}} e^{-\alpha_{f}\left|x_{3}-x_{3}^{\prime}\right|} & -i k_{\|} \operatorname{sgn}\left(x_{3}-x_{3}^{\prime}\right) e^{-\alpha_{f}\left|x_{3}-x_{3}^{\prime}\right|} \\
-i k_{\|} \operatorname{sgn}\left(x_{3}-x_{3}^{\prime}\right) e^{-\alpha_{f}\left|x_{3}-x_{3}^{\prime}\right|} & \alpha_{f} e^{-\alpha_{f}\left|x_{3}-x_{3}^{\prime}\right|}
\end{array}\right),
$$

where $\alpha_{f}^{2}=k_{\|}^{2}-\frac{\omega^{2}}{v_{f}^{2}}$, and

$$
G_{s}\left(x_{3}, x_{3}^{\prime}\right)=\left(\begin{array}{cc}
-\frac{k_{\|}^{2}}{2 \rho_{s} \omega^{2} \alpha_{\ell}}\left[e^{-\alpha_{\ell}\left|x_{3}-x_{3}^{\prime}\right|}-\zeta e^{-\alpha_{t}\left|x_{3}-x_{3}^{\prime}\right|}\right] & \frac{i k_{\|}}{2 \rho_{s} \omega^{2}} \operatorname{sgn}\left(x_{3}-x_{3}^{\prime}\right)\left[e^{-\alpha_{t}\left|x_{3}-x_{3}^{\prime}\right|}-e^{-\alpha_{\ell}\left|x_{3}-x_{3}^{\prime}\right|}\right] \\
\frac{i k_{\|}}{2 \rho_{s} \omega^{2}} \operatorname{sgn}\left(x_{3}-x_{3}^{\prime}\right)\left[e^{-\alpha_{t}\left|x_{3}-x_{3}^{\prime}\right|}-e^{-\alpha_{\ell}\left|x_{3}-x_{3}^{\prime}\right|}\right] & -\frac{k_{\|}^{2}}{2 \rho_{s} \omega^{2} \alpha_{t}}\left[e^{-\alpha_{t}\left|x_{3}-x_{3}^{\prime}\right|}-\zeta e^{-\alpha_{\ell}\left|x_{3}-x_{3}^{\prime}\right|}\right]
\end{array}\right),
$$

where

$$
\alpha_{t}^{2}=k_{\|}^{2}-\frac{\omega^{2}}{v_{t}^{2}}, \quad \alpha_{\ell}^{2}=k_{\|}^{2}-\frac{\omega^{2}}{v_{\ell}^{2}}, \quad \zeta=\frac{\alpha_{t} \alpha_{\ell}}{k_{\|}^{2}} .
$$

\section{Green's functions of fluid and solid layers in their space of interfaces}

The Green's functions of fluid and solid slabs in their space of interfaces $M=\left\{-d_{f} / 2,+d_{f} / 2\right\}$ and $\left\{-d_{s} / 2,+d_{s} / 2\right\}$ are given by ${ }^{53,54}$

$$
g_{f}^{-1}(M M)=\left(\begin{array}{cccc}
0 & 0 & 0 & 0 \\
0 & a & 0 & b \\
0 & 0 & 0 & 0 \\
0 & b & 0 & a
\end{array}\right)
$$

where

$$
\begin{gathered}
a=\frac{\rho_{f} \omega^{2} \cosh \left(\alpha_{f} d_{f}\right)}{\alpha_{f} \sinh \left(\alpha_{f} d_{f}\right)}, \\
b=-\frac{\rho_{f} \omega^{2}}{\alpha_{f} \sinh \left(\alpha_{f} d_{f}\right)}
\end{gathered}
$$

with

$$
\begin{gathered}
\alpha_{f}^{2}=k_{\|}^{2}-\frac{\omega^{2}}{v_{f}^{2}}, \\
g_{s}^{-1}(M M)=\left(\begin{array}{cccc}
h & i q & d & \text { if } \\
-i q & r & i f & e \\
d & -i f & h & -i q \\
-i f & e & i q & r
\end{array}\right),
\end{gathered}
$$

where

$$
h=\frac{N \alpha_{\ell} \omega^{2}}{2 k_{\|} v_{t}^{2}}\left[\sinh \left(\alpha_{t} d_{s}\right) \cosh \left(\alpha_{\ell} d_{s}\right)-\zeta \sinh \left(\alpha_{\ell} d_{s}\right) \cosh \left(\alpha_{t} d_{s}\right)\right],
$$

$$
r=\frac{N \alpha_{t} \omega^{2}}{2 k_{\|} v_{t}^{2}}\left[\sinh \left(\alpha_{\ell} d_{s}\right) \cosh \left(\alpha_{t} d_{s}\right)-\zeta \sinh \left(\alpha_{t} d_{s}\right) \cosh \left(\alpha_{\ell} d_{s}\right)\right],
$$

$$
\begin{aligned}
q= & N\left\{\zeta ( 3 k _ { \| } ^ { 2 } + \alpha _ { t } ^ { 2 } ) \left[\sinh ^{2}\left(\alpha_{\ell} \frac{d_{s}}{2}\right) \cosh ^{2}\left(\alpha_{t} \frac{d_{s}}{2}\right)\right.\right. \\
& \left.+\sinh ^{2}\left(\alpha_{t} \frac{d_{s}}{2}\right) \cosh ^{2}\left(\alpha_{\ell} \frac{d_{s}}{2}\right)\right] \\
& \left.-\frac{1}{2}\left[2 \alpha_{\ell} \alpha_{t} \zeta+\left(k_{\|}^{2}+\alpha_{t}^{2}\right)\right] \sinh \left(\alpha_{\ell} d_{s}\right) \sinh \left(\alpha_{t} d_{s}\right)\right\},
\end{aligned}
$$

$$
d=-\frac{N \alpha_{\ell} \omega^{2}}{2 k_{\|} v_{t}^{2}}\left[\sinh \left(\alpha_{t} d_{s}\right)-\zeta \sinh \left(\alpha_{\ell} d_{s}\right)\right]
$$

$$
\begin{gathered}
e=-\frac{N \alpha_{t} \omega^{2}}{2 k_{\|} v_{t}^{2}}\left[\sinh \left(\alpha_{\ell} d_{s}\right)-\zeta \sinh \left(\alpha_{t} d_{s}\right)\right], \quad \text { (A12 } \\
f=-N\left[2 \alpha_{\ell} \alpha_{t}-\zeta\left(k_{\|}^{2}+\alpha_{t}^{2}\right)\right]\left[\sinh ^{2}\left(\alpha_{\ell} \frac{d_{s}}{2}\right)-\sinh ^{2}\left(\alpha_{t} \frac{d_{s}}{2}\right)\right]
\end{gathered}
$$

with

$$
\begin{aligned}
N= & -\frac{\rho_{s} v_{t}^{2}}{2 k_{\|}}\left[\cosh \left(\alpha_{\ell} \frac{d_{s}}{2}\right) \sinh \left(\alpha_{t} \frac{d_{s}}{2}\right)\right. \\
& \left.-\zeta \cosh \left(\alpha_{t} \frac{d_{s}}{2}\right) \sinh \left(\alpha_{\ell} \frac{d_{s}}{2}\right)\right]^{-1} \\
& \times\left[\sinh \left(\alpha_{\ell} \frac{d_{s}}{2}\right) \cosh \left(\alpha_{t} \frac{d_{s}}{2}\right)\right. \\
& \left.-\zeta \sinh \left(\alpha_{t} \frac{d_{s}}{2}\right) \cosh \left(\alpha_{\ell} \frac{d_{s}}{2}\right)\right]^{-1}
\end{aligned}
$$

and

$$
\alpha_{t}^{2}=k_{\|}^{2}-\frac{\omega^{2}}{v_{t}^{2}}, \quad \alpha_{\ell}^{2}=k_{\|}^{2}-\frac{\omega^{2}}{v_{\ell}^{2}}, \quad \zeta=\frac{\alpha_{t} \alpha_{\ell}}{k_{\|}^{2}} .
$$


${ }^{1}$ L. M. Brekhovskikh, Waves in Layered Media (Academic, New York, 1981).

${ }^{2} \mathrm{P}$. Yeh, Optical Waves in Layered Media (Wiley, New York, 1988).

${ }^{3}$ B. Djafari-Rouhani and J. Sapriel, Surf. Sci. Rep. 10, 189 (1989).

${ }^{4} \mathrm{~S}$. Tamura, Proceedings of the 6th International Conference on Phonon Scattering in Condensed Matter, Heidelberg, 1989 (World Scientific, Singapore, 1990), p. 703.

${ }^{5}$ D. J. Lockwood and J. F. Young, Light Scattering in Semiconductor Structures and Superlattices (Plenum, New York, 1991).

${ }^{6}$ B. Jusserand and M. Cardona, in Light Scattering in Solids, edited by M. Cardona and G. Güntherodt (Springer, Berlin, 1989), p. 49.

${ }^{7}$ S. I. Tamura, Phys. Rev. B 39, 1261 (1989); S. Mizuno and S. I. Tamura, ibid. 45, 13423 (1992).

${ }^{8}$ E. H. El Boudouti, B. Djafari-Rouhani, E. M. Khourdifi, and L. Dobrzynski, Phys. Rev. B 48, 10987 (1993); E. H. El Boudouti, B. Djafari-Rouhani, A. Akjouj, and L. Dobrzynski, ibid. 54, 14728 (1996).

${ }^{9}$ F. Garcia-Moliner and V. R. Velasco, Theory of Single and Multiple Interfaces. The Method of Surface Green Function Matching (World Scientific, Singapore, 1992).

${ }^{10}$ A. S. Barker, J. L. Merz, and A. C. Gossard, Phys. Rev. B 17, 3181 (1978).

${ }^{11}$ V. Narayanamurti, H. L. Stormer, M. A. Chin, A. C. Gossard, and W. Wiegmann, Phys. Rev. Lett. 43, 2012 (1979).

${ }^{12}$ O. Koblinger, J. Mebert, E. Dittrich, S. Dottinger, W. Eisenmenger, P. V. Santos, and L. Ley, Phys. Rev. B 35, 9372 (1987).

${ }^{13}$ D. J. Dieleman, A. F. Koenderink, M. G. A van Veghel, A. F. M. Arts, and H. W. de Wijn, Phys. Rev. B 64, 174304 (2001).

${ }^{14}$ S. Tamura, D. C. Hurley, and J. P. Wolfe, Phys. Rev. B 38, 1427 (1988).

${ }^{15}$ C. Colvard, R. Merlin, M. V. Klein, and A. C. Gossard, Phys. Rev. Lett. 45, 298 (1980).

${ }^{16}$ B. Jusserand, D. Paquet, F. Mollot, F. Alexandre, and G. LeRoux, Phys. Rev. B 35, 2808 (1987).

${ }^{17}$ D. J. Lockwood, R. L. S. Devine, A. Rodriguez, J. Mendialdua, B. Djafari-Rouhani, and L. Dobrzynski, Phys. Rev. B 47, 13553 (1993).

${ }^{18}$ P. J. Shull, D. E. Chimenti, and A. Safaeinili, J. Acoust. Soc. Am. 95, 99 (1994); A. Safaeinili and D. E. Chimenti, ibid. 98, 2336 (1995).

${ }^{19}$ H. T. Grahn, H. J. Maris, J. Tauc, and B. Abeles, Phys. Rev. B 38, 6066 (1988).

${ }^{20}$ A. Yamamoto, T. Mishina, Y. Masumoto, and M. Nakayama, Phys. Rev. Lett. 73, 740 (1994).

${ }^{21}$ A. Bartels, T. Dekorsy, and H. Kurz, Appl. Phys. Lett. 72, 2844 (1998); A. Bartels, T. Dekorsy, H. Kurz, and K. Köhler, Phys. Rev. Lett. 82, 1044 (1999).

${ }^{22}$ P. Sondhauss, J. Larsson, M. Harbst, G. A. Naylor, A. Plech, K. Scheidt, O. Synnergren, M. Wulff, and J. S. Wark, Phys. Rev. Lett. 94, 125509 (2005).

${ }^{23}$ B. Djafari-Rouhani, L. Dobrzynski, O. Hardouin Duparc, R. E. Camley, and A. A. Maradudin, Phys. Rev. B 28, 1711 (1983).

${ }^{24}$ T. Aono and S. I. Tamura, Phys. Rev. B 58, 4838 (1998).

${ }^{25}$ S. Mizuno and S. I. Tamura, Phys. Rev. B 53, 4549 (1996).

${ }^{26}$ M. Hammouchi, E. H. El Boudouti, A. Nougaoui, B. DjafariRouhani, M. L. H. Lahlaouti, A. Akjouj, and L. Dobrzynski, Phys. Rev. B 59, 1999 (1999).

${ }^{27}$ H. J. Trodahl, P. V. Santos, G. V. M. Williams, and A. Bittar,
Phys. Rev. B 40, R8577 (1989).

${ }^{28}$ W. Chen, Y. Lu, H. J. Maris, and G. Xiao, Phys. Rev. B 50, 14506 (1994).

${ }^{29}$ B. Perrin, B. Bonello, J. C. Jeannet, and E. Romatet, Physica B 219\&220, 681 (1996); B. Bonello, B. Perrin, E. Romatet, and J. C. Jeannet, Ultrasonics 35, 223 (1997).

${ }^{30}$ N.-W. Pu and J. Bokor, Phys. Rev. Lett. 91, 076101 (2003); N.-W. Pu, Phys. Rev. B 72, 115428 (2005).

${ }^{31}$ E. M. Khourdifi and B. Djafari-Rouhani, Surf. Sci. 211-212, 361 (1989).

${ }^{32}$ D. Bria, E. H. El Boudouti, A. Nougaoui, B. Djafari-Rouhani, and V. R. Velasco, Phys. Rev. B 60, 2505 (1999).

${ }^{33}$ E. M. Khourdifi and B. Djafari-Rouhani, J. Phys.: Condens. Matter 1, 7543 (1989).

${ }^{34}$ D. Bria, E. H. El Boudouti, A. Nougaoui, B. Djafari-Rouhani, and V. R. Velasco, Phys. Rev. B 61, 15858 (2000).

${ }^{35}$ K.-Q. Chen, X.-H. Wang, and B.-Y. Gu, Phys. Rev. B 61, 12075 (2000).

${ }^{36}$ S. Mizuno, Phys. Rev. B 65, 193302 (2002).

${ }^{37}$ S. I. Tamura, H. Watanabe, and T. Kawasaki, Phys. Rev. B 72, 165306 (2005).

${ }^{38}$ G. P. Schwartz, G. J. Gualtieri, and W. A. Sunder, Appl. Phys. Lett. 58, 971 (1991).

${ }^{39}$ M. Trigo, A. Bruchhausen, A. Fainstein, B. Jusserand, and V. Thierry-Mieg, Phys. Rev. Lett. 89, 227402 (2002); P. Lacharmoise, A. Fainstein, B. Jusserand, and V. Thierry-Mieg, Appl. Phys. Lett. 84, 3274 (2004); N. D. Lanzillotti Kimura, A. Fainstein, and B. Jusserand, Phys. Rev. B 71, 041305(R) (2005).

${ }^{40}$ D. Bria and B. Djafari-Rouhani, Phys. Rev. E 66, 056609 (2002), and references therein.

${ }^{41}$ B. Manzanares-Martinez, J. Sanchez-Dehesa, A. Hakansson, F. Cervera, and F. Ramos-Mendieta, Appl. Phys. Lett. 85, 154 (2004).

${ }^{42}$ See, for example, M. S. Kushwaha, P. Halevi, L. Dobrzynski, and B. Djafari-Rouhani, Phys. Rev. Lett. 71, 2022 (1993); M. Sigalas and E. N. Economou, Solid State Commun. 86, 141 (1993); F. R. Montero de Espinosa, E. Jimenez, and M. Torres, Phys. Rev. Lett. 80, 1208 (1998); J. O. Vasseur, P. A. Dymier, G. Frantziskonis, G. Hong, B. Djafari-Rouhani, and L. Dobrzynski, J. Phys.: Condens. Matter 10, 6051 (1998). I. E. Psarobas, N. Stefanou, and A. Modinos, Phys. Rev. B 62, 278 (2000); 62, 5536 (2000); S. Yang, J. H. Page, Z. Liu, M. L. Cowan, C. T. Chan, and P. Sheng, Phys. Rev. Lett. 88, 104301 (2002).

${ }^{43}$ A. Bousfia, Ph.D. thesis, University Mohamed I, Oujda, Morocco (2004)

${ }^{44}$ S. M. Rytov, Akust. Zh. 2, 71 (1956) [Sov. Phys. Acoust. 2, 68 (1956)].

${ }^{45}$ M. Schoenberg, Wave Motion 6, 303 (1984).

${ }^{46}$ M. Rousseau, J. Acoust. Soc. Am. 86, 2369 (1989).

${ }^{47}$ M. A. Biot, J. Acoust. Soc. Am. 28, 168 (1962).

${ }^{48}$ T. J. Plona, K. W. Winkler, and M. Schoenberg, J. Acoust. Soc. Am. 81, 1227 (1987).

${ }^{49}$ C. Gazanhes and J. Sageloli, Acustica 81, 221 (1995).

${ }^{50}$ R. James, S. M. Woodley, C. M. Dyer, and F. Humphrey, J. Acoust. Soc. Am. 97, 2041 (1995).

${ }^{51}$ M. Shen and W. Cao, Appl. Phys. Lett. 75, 3713 (1999).

${ }^{52}$ L. Dobrzynski, Surf. Sci. Rep. 11, 139 (1990).

${ }^{53}$ E. H. El Boudouti, Ph.D. thesis, University Mohamed I, Oujda, Morocco, 1998.

${ }^{54}$ L. Dobrzynski, J. Mendialdua, A. Rodriguez, S. Bolibo, and M. 
More, J. Phys. (France) 50, 2563 (1989).

${ }^{55}$ H. Kato, Phys. Rev. B 59, 11136 (1999); S. Mizuno, ibid. 63, 035301 (2001); B. Manzanares-Martinez and F. RamosMendieta, ibid. 66, 092302 (2002).

${ }^{56}$ A. Safaeinli and D. E. Chimenti, J. Acoust. Soc. Am. 98, 2336 (1995); H. Zhang, D. E. Chimenti, and S. Zeroug, ibid. 104, 1982 (1998); O. Lenoir, J. L. Izbicki, P. Rembert, G. Maze, and J. Ripoche, ibid. 9, 601 (1991).

${ }^{57}$ M. L. H. Lahlaouti, A. Akjouj, B. Djafari-Rouhani, L. Dobrzynski, M. Hammouchi, E. H. El Boudouti, A. Nougaoui, and B.
Kharbouch, Phys. Rev. B 63, 035312 (2001).

${ }^{58}$ J. Laperre and W. Thys, J. Acoust. Soc. Am. 92, 909 (1992).

${ }^{59}$ D. E. Chimenti and A. Nayfeh, J. Acoust. Soc. Am. 85, 555 (1988).

${ }^{60}$ CRC Handbook of Chemistry and Physics, 66th ed. (CRC, Boca Raton, FL, 1985), p. E-43.

${ }^{61}$ U. Fano, Phys. Rev. 124, 1866 (1961).

${ }^{62}$ B. Manzanares-Martinez and F. Ramos-Mendieta, Phys. Rev. B 61, 12877 (2000). 